\title{
Recent Advances in Intelligent Source Code Generation: A Survey on Natural Language Based Studies
}

\author{
Chen Yang $\mathbb{D}$, Yan Liu * and Changqing Yin \\ School of Software Engineering, Tongji University, Shanghai 201804, China; yangchensa@tongji.edu.cn (C.Y.); \\ yinchangqing@tongji.edu.cn (C.Y.) \\ * Correspondence: yanliu.sse@tongji.edu.cn
}

Citation: Yang, C.; Liu, Y.; Yin, C. Recent Advances in Intelligent Source Code Generation: A Survey on Natural Language Based Studies. Entropy 2021, 23, 1174. https:// doi.org/10.3390/e23091174

Academic Editor: Karsten Keller

Received: 13 July 2021

Accepted: 30 August 2021

Published: 7 September 2021

Publisher's Note: MDPI stays neutral with regard to jurisdictional claims in published maps and institutional affiliations.

Copyright: (c) 2021 by the authors. Licensee MDPI, Basel, Switzerland. This article is an open access article distributed under the terms and conditions of the Creative Commons Attribution (CC BY) license (https:// creativecommons.org/licenses/by/ $4.0 /)$.

\begin{abstract}
Source Code Generation (SCG) is a prevalent research field in the automation software engineering sector that maps specific descriptions to various sorts of executable code. Along with the numerous intensive studies, diverse SCG types that integrate different scenarios and contexts continue to emerge. As the ultimate purpose of SCG, Natural Language-based Source Code Generation (NLSCG) is growing into an attractive and challenging field, as the expressibility and extremely high abstraction of the input end. The booming large-scale dataset generated by open-source code repositories and Q\&A resources, the innovation of machine learning algorithms, and the development of computing capacity make the NLSCG field promising and give more opportunities to the model implementation and perfection. Besides, we observed an increasing interest stream of NLSCG relevant studies recently, presenting quite various technical schools. However, many studies are bound to specific datasets with customization issues, producing occasional successful solutions with tentative technical methods. There is no systematic study to explore and promote the further development of this field. We carried out a systematic literature survey and tool research to find potential improvement directions. First, we position the role of NLSCG among various SCG genres, and specify the generation context empirically via software development domain knowledge and programming experiences; second, we explore the selected studies collected by a thoughtfully designed snowballing process, clarify the NLSCG field and understand the NLSCG problem, which lays a foundation for our subsequent investigation. Third, we model the research problems from technical focus and adaptive challenges, and elaborate insights gained from the NLSCG research backlog. Finally, we summarize the latest technology landscape over the transformation model and depict the critical tactics used in the essential components and their correlations. This research addresses the challenges of bridging the gap between natural language processing and source code analytics, outlines different dimensions of NLSCG research concerns and technical utilities, and shows a bounded technical context of NLSCG to facilitate more future studies in this promising area.
\end{abstract}

Keywords: natural language-based source code generation; systematic literature review; machine learning application

\section{Introduction}

As the cost of data ingestion, storage and computation continues to decrease, applying AI in practice is becoming the focus of the whole IT industry. The enormous potential is unleashed with a combination of AI and other fields, and Source Code Generation (SCG) is no exception. Various deep learning algorithms and neural network construction approaches have been applied to the automatic SCG and intelligent software development environment. SCG allows practitioners to generate source code from a higher level of abstraction [1]. Processing methods more intelligent thatn SCG are opening new possibilities for the input abstraction levels, resulting in the endless emergence of new perspectives. Popular perspectives represented by specific descriptions can be instantiated as domain-specific language, formal language, modeling language, natural requirements, etc. Among these, 
natural requirements, including natural language or prototypes, have attracted intense academic interest due to their intuitiveness, roughness, and primitiveness.

As the ideal scenario for automatic SCG, research on Natural Language-based Source Code Generation (NLSCG) is booming. Some encouraging progress has been made, developing promising solutions with a confident performance. The majority of these studies start with problems of individuation coupled with specific datasets, presenting the tendency of tentative occasional successful solutions. There is no systematic study to promote the further development of this field. To understand the practitioners interested in NLSCG from their perspective, we brainstormed and sketched out an empathy map (see Figure 1) to direct the following research concerns and trends. As the empathy map expressed, participators, including researchers for typical NLSCG models, open-source community contributors for NLSCG products and toolchains, and automated software platform users for considering the degree of automation, are passionate about the NLSCG field. Under the mainstream trend of vigorous development of NLSCG, participants are curious about the baseline and the state-of-the-art NLSCG models.

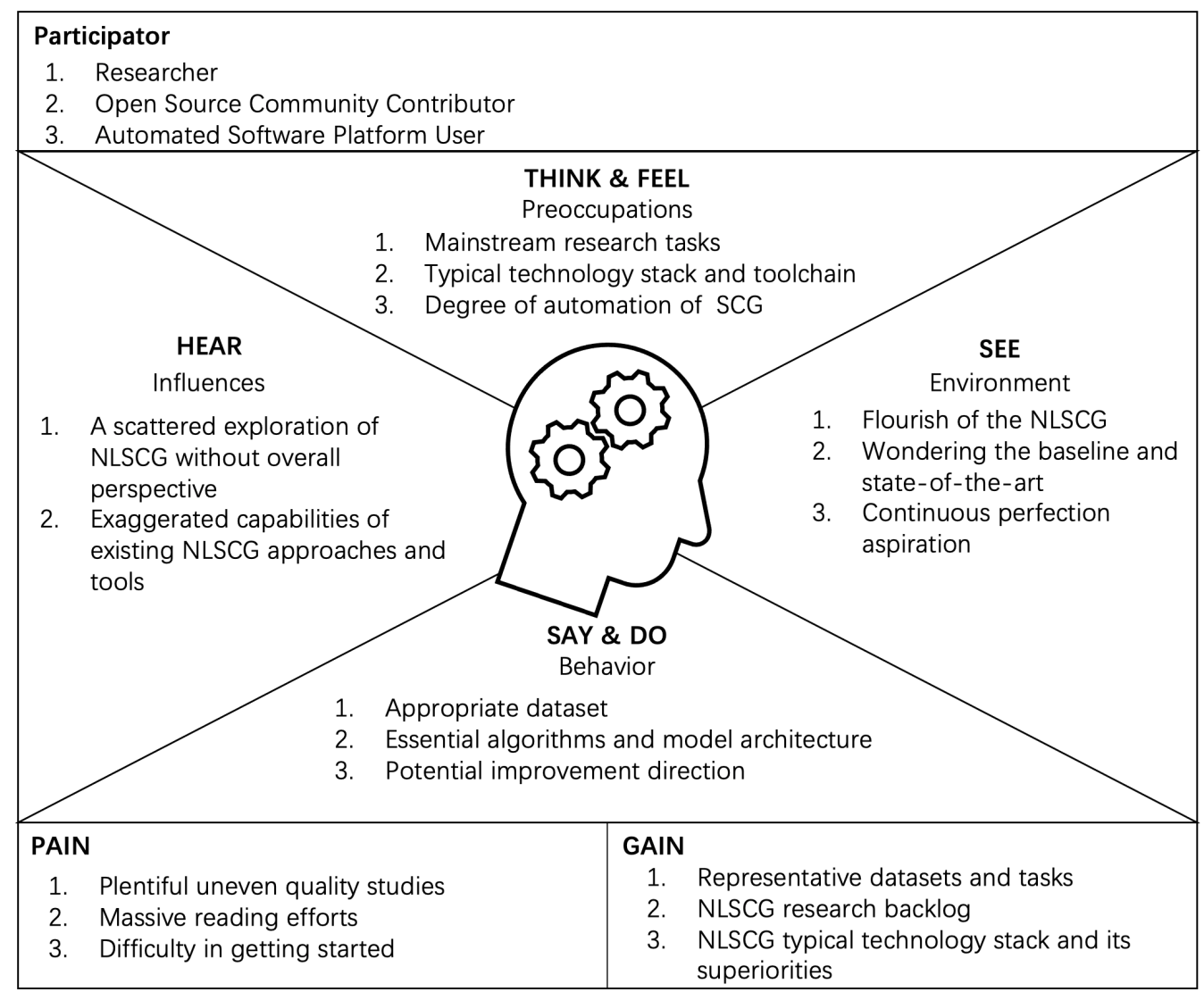

Figure 1. NLSCG empathy map.

However, the abundant relevant studies and tools driving the process make it more laborious and challenging for participants to obtain comprehensive and detailed NLSCG research actualities. They confront bad quality studies and tools, and have to devote massive amounts of effort to reading; hence, there are difficulties in quickly getting started. Trying to discern the outstanding direction of improvement from partial studies calls to mind the story of the blind men and the elephant. The improvements may inadvertently fall into a futile pitfall, so it is meaningless to promote the entire field. It is urgent to clarify the current development status of NLSCG, namely, the appropriate dataset, the essential algorithms and representative architecture of the transformation model, the living bottlenecks, the enabling factors, and the potential perfection directions. Based on these motivations, we investigate the actualities and future trends, summarize representative datasets and tasks, 
and gain insights from the research backlog of NLSCG. Finally, the NLSCG landscape depicts typical components, their correlations, and their advantages.

NLSCG allows users to generate source code from an extremely intuitive and primitive perspective. This end-to end manner is amicable for those who lack prior knowledge in source code. NLSCG processing techniques can also be applied to commercial automated software platforms, enabling us to establish natural language interfaces with executable source code, which can reduce the cost of learning and the training cost to a certain extend. Research in this field shows its significance, as the learned experiences would be applied to similar scenarios that transform from abstract and fuzzy descriptions to highly structural constrained representations. In addition, widespread application of deep learning has promoted the prosperity of the transformation between natural language and source code like semantic code search [2] and automatic comment generation [3]. However, natural language precisely challenges the efficient construction of transformation models due to its ambiguity and uncertainty. The well formedness, type-sensitiveness, executability [4], and semantics of source code create the most difficulties. Many studies [4-69] have been conducted on the NLSCG and its relevant tasks by employing baseline and state-of-the-art deep learning models and algorithms. Abundant relevant studies are creating momentum to make this the right moment to explore the entire NLSCG field, with the expectation of discovering its actualities, potentials, and improvements.

This paper makes the following contributions: (1) We position the role of NLSCG and specify the context among various SCG genres empirically based on the domain knowledge and programming considerations. (2) We clarify the NLSCG field via an elaborate literature survey and get a distinct problem understanding. (3) We model the NLSCG research problems by explicating technical focus and adaptive challenges and gaining insights from the research backlog. (4) We summarize the technology landscape of NLSCG through exhaustive research on the transformation model and depict the critical tactics used in the essential components and their correlations.

The remainder of this paper is organized as follows. We introduce our research journey which guides our subsequent works in Section 2. In Section 3, various code generation genres are introduced before we derive a definition of NLSCG. Section 4 strengthens the process of NLSCG problem understanding through a systematic literature tracking approach. We elaborate on the insights from the research backlog in Section 5. In Section 6, the state-of-art NLSCG technology landscape and essential components along the generating pipeline are depicted, and this survey is concluded in Section 7.

\section{Research Journey and Context}

Figure 2 presents the research journey we followed in this survey. The journey starts with explaining the various SCG genres to clearly examine the research vision, in which we position the role of NLSCG among these genres. To figure out the state-of-the-art and potentials of NLSCG exhaustively, we launched an exploration via three phases: (1) Problem understanding; (2) research backlog; and (3) utilities. The three phases are present in succession; the conclusion obtained by the former is considered as the basis and constraints for the latter, while the latter echoes the former.

Problem Positioning: Various SCG genres constantly emerge and develop, contributing to the building of the software engineering automation ecosystem. Gazing at the ecosystem from the overall perspective, we specify the NLSCG context, expanding the entire research vision, which implicates specific impacts on NLSCG research concerns and technical means. Based on the understanding and analysis of automation software engineering, we position the role of NLSCG among the various SCG genres in terms of the application scenarios, the input and output style, the conversion direction, and the popularity, and finally propose a preliminary definition of NLSCG. 


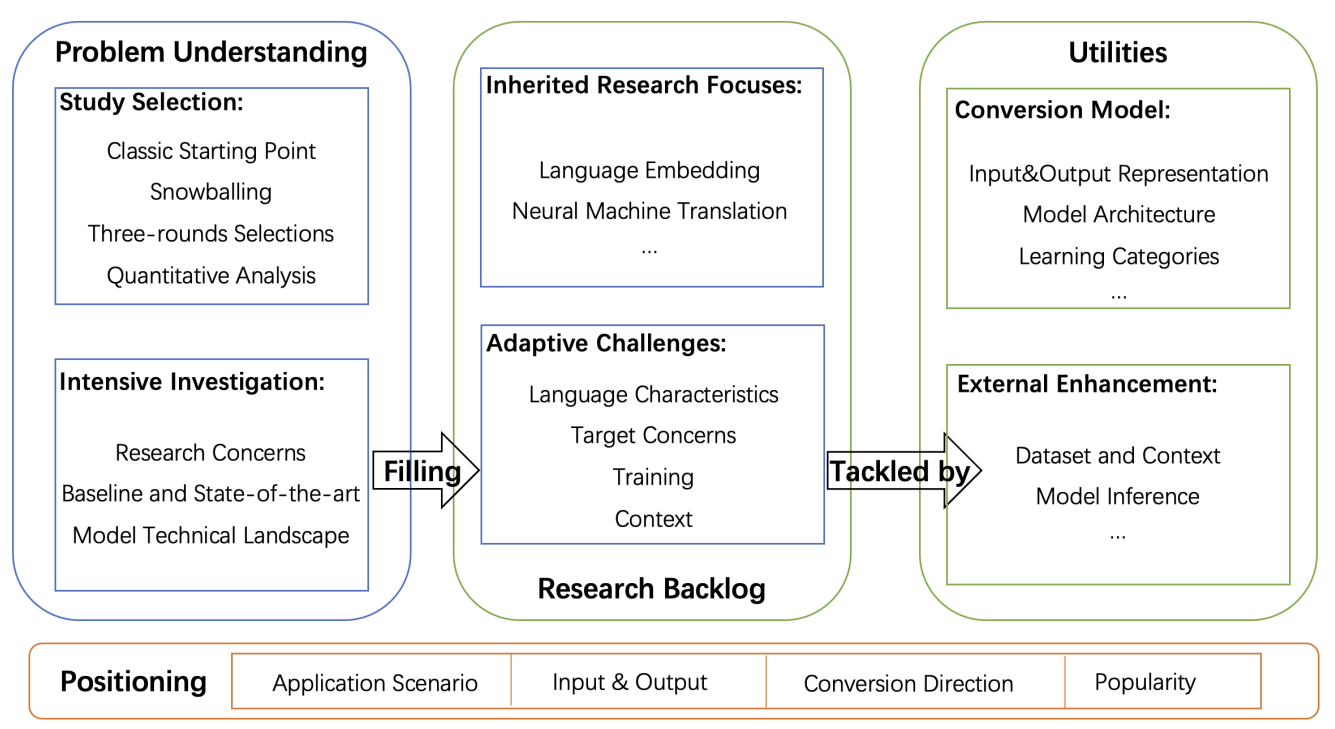

Figure 2. The Research Journey for this Survey.

Problem Understanding: We leverage the snowballing literature collection method and its matched analysis to enhance the problem understanding. NLSCG possesses a small domain scope and presents a certain homogeneity technical tendency. The very classic and well-known studies in the NLSCG field are selected before the snowballing process is executed. Afterward, depending on the predefined selection rules (expected to be three-round selections), the literature snowballing continuously until the appropriate significant studies are screened out. Next, we perform quantitative analysis to get critical statistical information from these potential studies. After completing these peripheral tasks, we investigate these selected studies intensively with specific purposes, laying a foundation for our subsequent works. These purposes include exploring research concerns, technical landscape, baseline, and state-of-the-art model details. We abstract the crude prototype of NLSCG backlog items from the research concerns and summarize the NLSCG utilities from model-relevant attempts. We build up a deepening understanding of the NLSCG research context from these investigations, and it paves the way for better exploration and summarization of our subsequent steps.

Research Backlog: To identify the ongoing concerns of the NLSCG field and consequently understand current efforts and potential directions of future improvement, we present the research backlog for a more vivid expression. Adapting the term "backlog" coined from agile development, we consider it the "research to-do task" in this NLSCG field. Through series of readings on the selected studies collected by the snowballing process, we accumulate the research backlog in terms of resource, environment, boundary, enabler, utilities, etc., with the research concerns as a clue. We tentatively extract a taxonomy based on scattered research backlog items from the perspectives of inherited research focuses and adaptive challenges. While this "taxonomy" exhibits immaturity and fragility, it can be regarded as a feasible scheme to collect the research backlog items. Finally, we summarize insights gained from the NLSCG research backlog and elaborate the details in conjunction with specific studies. The research backlog deepens the understanding of the NLSCG problem and contributes to sketching up the essential utilities for NLSCG.

Utilities: As the critical part of the NLSCG solutions, the design and technical implementation details of the transformation models have shown steady attraction for relevant practitioners. Several aspects are particularly remarkable, including the baseline and essential components indicating how the model sprouts; how the advanced models are explored from different angles; how the fuzzy mappings between research concerns and implementations function; and the tactics adopted by every pivotal component. Similarly, we gain much raw information about NLSCG model attempts based on the selected studies from the snowballing. Taking the research backlog as a guideline, we decompose the raw 
information, convert it into essential components, and then build interactions according to the data processing chain and the conventional deep learning model pipeline. Finally, we summarize and depict all critical tactics and give a detailed explanation of the transformation model, and we regard them as the technical utilities of NLSCG. The utilities can tackle the current backlog items from two directions: Internal model advancement and external enhancement. Apparently, advancements deriving from the transformation model, such as input and output representation, model architecture, learning categories, etc., are worth great effort. Besides, embracing diverse and divergent thinking upon these predefined directions until covering all mainstream processing manners is also an indispensable part of investigating the essential utilities. Some studies go in the opposite direction, searching for probable routes of external enhancement, such as the dataset quality that determines the upper-bounds of the model performance, the retrieval strategy in the model inference stage, etc. The essential utilities would bring a comprehensive understanding of NLSCG models to beginners, and, what is more, it would inspire practitioners with more potential improvements in this field.

\section{Source Code Generation Genres}

Various description systems are proposed to depict, design, and refine software objects and artifacts, and these description systems are then considered as the input end of SCG. Automatic generating source code from various description styles is a historical field both in academic and industrial areas. Limited by the characteristics of the description system, different approaches present various tendencies. According to automation software engineering status, the dominating SCG genres and their correlations are shown in Figure 3. Overall, the abstract level of description increases from the direction of right-to-left in the pipeline. The farther the horizontal distance between the two description systems in Figure 3, the more difficult it is to convert between them due to their vast degree of abstraction. Different SCG genres show their unique application scenario and popularity and are coupled with the technical conditions at the time. Some genres are still active, like NRA, DSLA, and GMA, while others are gradually lacking attention like FLA and EMA. Besides, the transformations between different description systems also attract a certain amount of attention, such as code summarization, reverse engineering, etc. We summarized the primary SCG genres as follows.

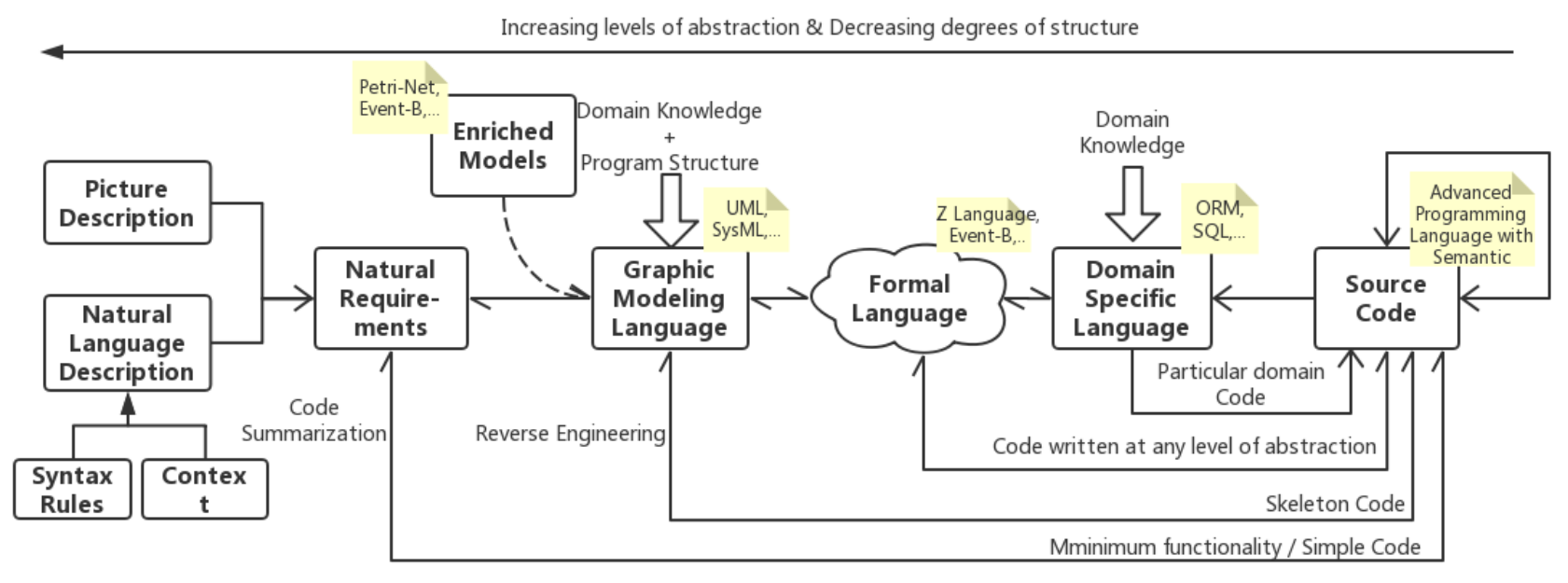

Figure 3. Source Code Generation Genres.

Domain-Specific Language based Approach (DSLA): As the most commonly applied approach in the industry, the transformation between domain-specific language (DSL) and source code tends to be business friendly and to involve close-domain couplings. DSL specifies a set of domain-related rules that the software must obey and run on a dedicated, high-reliability scenario [70], presenting extreme precision of transformation. This would achieve more cost effectiveness during the development process. From another perspective, 
the transformation is driven by rules manually defined by domain experts and would bring a higher cost of learning in the initial stage.

Formal Language based Approach (FLA): The formal method describes and analyzes the behavior of software systems utilizing mathematical symbols, and the verifiability is capable of improving the correctness of the system [71]. FLA possesses high accuracy under specific domains and fits relatively common scenarios. However, formal language-based methods and techniques raise a high demand for practitioners, resulting in abundant learning and training efforts to adapt to the rigid limitation. They have to make trade-offs between the production cost and effectiveness, and this limitation narrows the scope of application in the industry area.

Graphic Modeling-based Approach (GMA): Generating source code from modeling languages supporting object-oriented analysis and design is a classical direction. This approach focuses on the high-level design of systems, for instance, the static skeleton code (like class declaration, function definition, etc.) of a specific scenario. Relatively complex research can produce the business process as well [72]. The main challenge in GMA is to replenish the details of the generated code [73]. GMA possesses a more substantial universality independent of implementation details and could adapt to various software development scenarios. However, conversions between the two highly abstract description systems will lead to unavoidable information loss. In addition, GMA pays close attention to static mapping, and the generated source code with the simple logic flow cannot recreate the details, making it infeasible to be applied in practical application.

Enriched Models based Approach (EMA): It has been a long time since UML has been applied to design object-oriented systems. However, the ambiguity of models created by UML specifications makes it inappropriate for it to be the starting point of automatic code generation [74]. Models attached by strictly defined mathematical concepts and objects, such as Petri net, are promising directions for improvement. EMA focuses on the rigorous mapping of UML notations to the object-oriented class of enhanced models. While limited by the restricted structure of models and exponential efforts while constructing the reachability graphs, EMA can still greatly enhance the accuracy, readability, or extensibility of the generated code in some instances.

Nature Requirement based Approach (NRA): As continues on semantic parsing and deep learning, NRA has become one of the most active research topics in SCG. The natural requirement can be divided into prototype figure description [75] and natural language description. It is the most intuitive approach to generating source code from natural language, especially for programming beginners. NRA departs from past work on methodology and mode of thinking for the primitive, completely undesigned input end. At present, NRA tends to generate simple code snippets which implement minimum functionalities with unsteady performance. Researchers are trying to improve the transformation performance by attaching syntactic constraints and contextual information.

In the SCG genre diagram, various description forms and their conversion paths are highlighted. We describe the conversion direction, pros, and cons of each SCG genre in terms of the description systems features and research actualities. Each genre has its characteristics and has enlivened recent studies in certain areas, but they present as more outmoded than NRA. NRA, especially the natural language description-based approach, is the most popular, promising, and worthy of consideration. The challenges confronted by natural language-based source code generation (NLSCG) is foreseeable; we tentatively propose the definition of NLSCG as follows:

Given a specific problem context C ( $C$ can not be specified in the code snippet generation and program synthesis task types, refer to Section 4.3) and a natural language description NL to C, natural language-based source code generation (NLSCG) converts the input NL into the output executable source code (SC) corresponding to that NL. During the generation process, the semantic integrity of $\mathrm{NL}$, the well-formedness, type-sensitiveness, executability of SC, and the semantic consistency between the two ends must be guaranteed. 
In addition, when $C$ is given, NL must embed appropriate information from $C$, and the compatibility of SC and C must be guaranteed.

\section{Problem Understanding Process}

Abiding by the research journey map, the process started with well-designed research snowballing with the goal of acquiring valuable studies. We carried out the iterative forward and backward snowballing to obtain a mass of studies and employed three round screening to get small minority studies suitable for follow-up research. These selected studies are subsequently utilized for quantitative analysis to obtain preliminary viewpoints on recent trends in the NLSCG field. More importantly, these preliminary investigations have contributed to the refinement of NLSCG task types based on the basic NLSCG definition. There are slight differences between these types in research concerns and modeling priorities. Finally, we payed attention to the indispensable dataset for constructing the NLSCG models and summarized 36 datasets as well as their foundational information and applied task types.

\subsection{Snowballing Process}

Recently, an increasing number of studies have been published in the open-source database. To collect studies related to intelligent code generation and its similar tasks scientifically and effectively, we utilize the snowballing approach [76]. A very classic and well-known paper LPN [5] is selected as the starting point of the snowballing process. LPN proposes a novel NLSCG neural architecture considering the conditioning context and granularity of generation, and the creation of two new datasets, especially Hearthstone, has launched a new era for the NLSCG field.

The whole literature snowballing process and selection result sets are shown in Figure 4 . With the starting point of LPN, by utilizing forward snowballing, we collected 135 citations. In the next round of screening, 24 studies were retained after applying selection criteria by title, abstract, and full text by turns. Then we conducted backward snowballing. We collected 768 references and retained 24 studies after removing duplicates and screening by title, abstract, and full text by turns. After this round of screening, 48 studies were retained in total. To prevent possible omissions and obtain the latest results, we carried out a new round of forward snowballing and collected 3079 citations. Afterward, we conducted a round of screening in advance by Google Scholar relevance and timeliness for the sake of effectively filtering. Finally, through a full round of three-level screening, 66 studies were selected (the complete list of the selected studies is available online: Selected Studies List Ref: https:/ /github.com/Ada12/NLSCG_Research/blob/ main/selected-studies-list.md, accessed on 20 August 2021).

\subsection{Studies Analysis}

This section conducted a simple quantitative analysis of the selected studies from publication year, adopted datasets, and generation task dimensions. We summarized the development trend of automatic code generation in recent years and the most classic dataset classification through an intuitive two-dimensional diagram. As shown in Figure 5, the serial number in each grid represents the selected research ID, and the left half of the horizontal axis represents the publication year. The dataset type present on the right and the vertical axis represents the type of generation task described in Section 4.3. Besides, we summed up the utilized datasets by different studies to provide references for future readers. Section 4.4 elaborates the detail of all datasets used in selected studies.

Studies are classified into at least one code generation task type according to the adopted datasets and their introductions. The left half of Figure 5 shows the study IDs related to the four code generation task types in the past five years. Only one study was published in 2015, and since then the number of published studies has been increasing continuously, especially in the years 2018 and 2019. Due to the screening time range limitation, only a small part of the 2020 studies was selected. Some studies tend to research 
domain-general problems across different tasks, using multiple types of datasets. The most conspicuous representatives are studies 17 [6] and 18 [7], which employ various datasets across three task types.

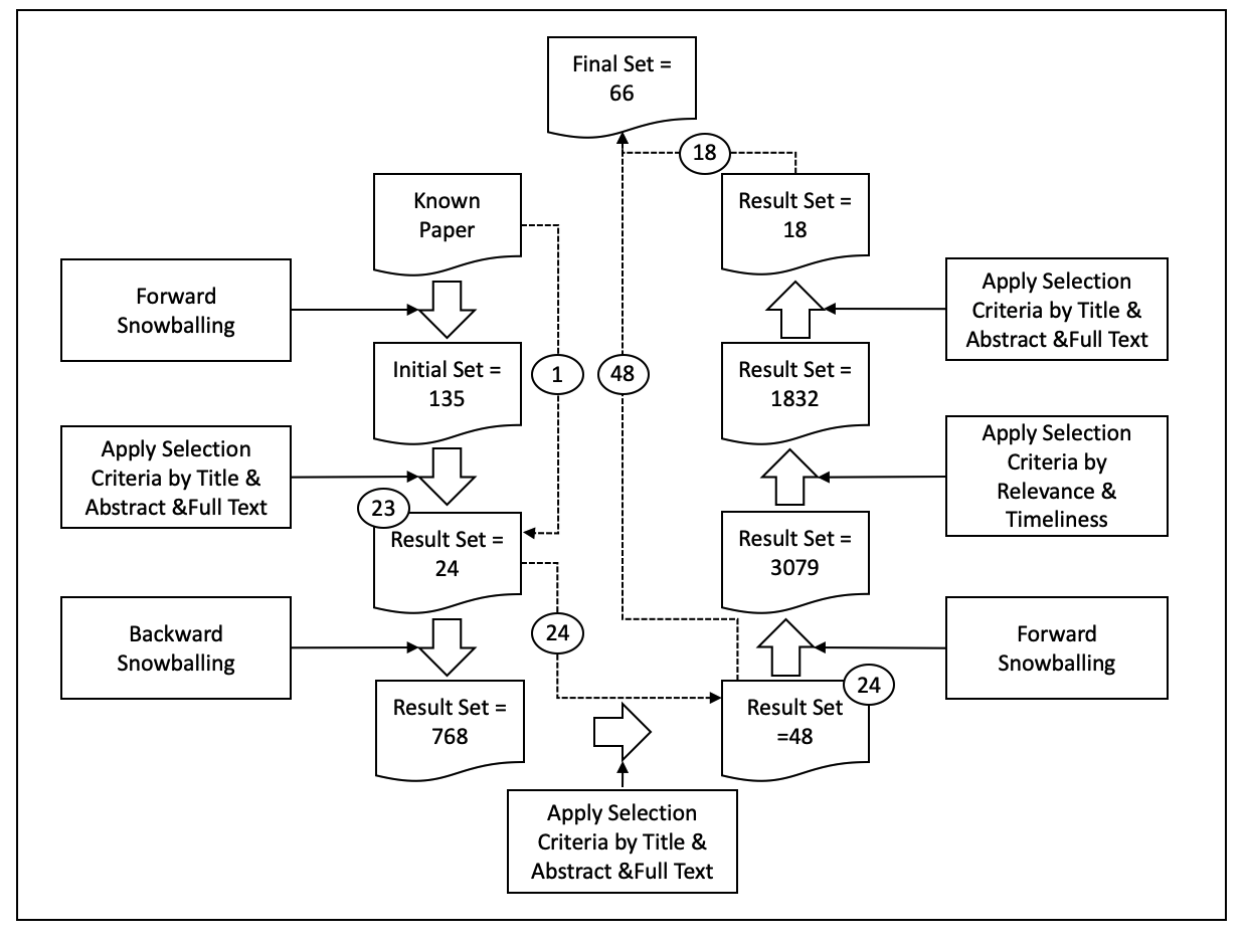

Figure 4. NLSCG Snowballing Process (until 31 May 2020).

\begin{tabular}{|c|c|c|c|c|c|}
\hline & & 6 & $9,15,45$ & $17,19,46$ & \\
\hline & 1 & 2,3 & $\begin{array}{c}8,10,12, \\
16\end{array}$ & $\begin{array}{c}11,17,18, \\
22,23,65\end{array}$ & \\
\hline & & $\begin{array}{c}32,34,35, \\
36,37,40\end{array}$ & $\begin{array}{c}7,10,21, \\
33,42,44, \\
54,55,56, \\
57,62\end{array}$ & $\begin{array}{c}18,24,48,56,5 \\
62,\end{array}$ & 51,53, \\
\hline 29 & $\begin{array}{c}5,25,26, \\
27,28,31\end{array}$ & $\begin{array}{c}3,4,13, \\
14,30,32, \\
37,39,61\end{array}$ & $\begin{array}{c}8,38,41, \\
43,44,49, \\
50,54,57\end{array}$ & $\begin{array}{c}11,17,18, \\
20,23, \\
46,48,58, \\
59,60,65\end{array}$ & 63 \\
\hline
\end{tabular}

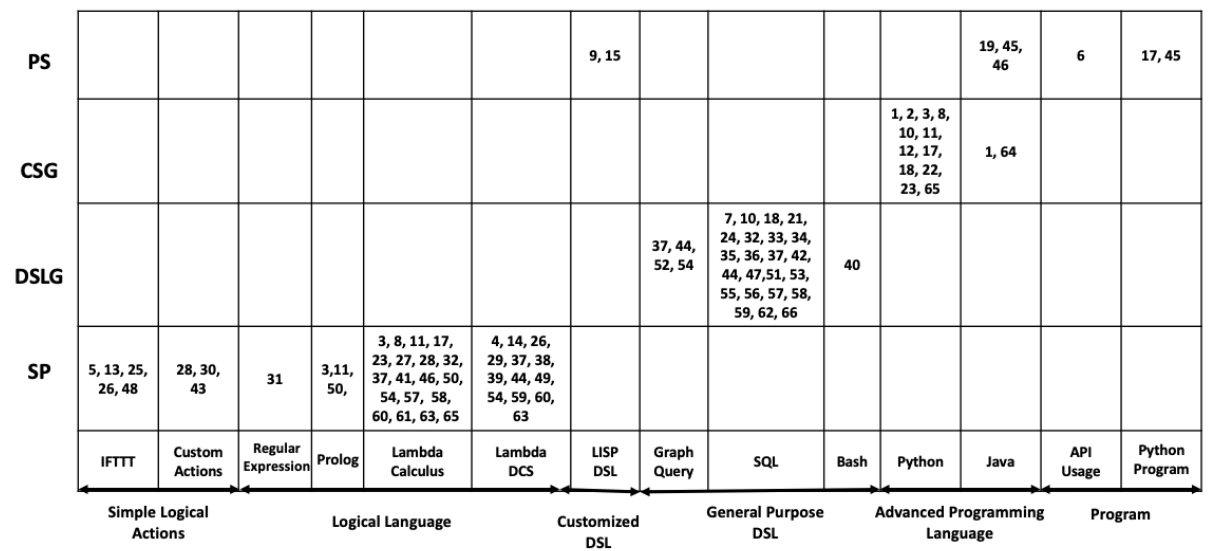

Figure 5. Selected Studies Distribution between Publication Year, Generation Task and Dataset.

The right half of Figure 5 shows the study IDs with specific generation task types and the target datasets. The output language corresponding to the dataset becomes increasingly complicated from the left-to-right direction. The complexity of syntax, the richness of semantic, the length of utterance-denotation pairs, and more importantly, the locality design pattern and the global architecture tactics make different ensemble generation languages with a qualitative leap. In general, to achieve the goal of code generation tasks, various datasets are proposed as the basis for training in this area. The lambda calculus and its variants lambda DCS, SQL, and Python are the most popular generation style. It is worth noting that program synthesis takes customized DSL [8,9] as a springboard for better bridging the huge abstraction degree gap between natural language and programs. 


\subsection{Natural Language-Based Intelligent Code Generation Tasks}

Considering SCG tasks as machine translation [77] has kicked off a generation storm tide by introducing deep learning to this field. Deep learning has gradually become the most commonly used technology for such tasks, and impressive performance has been achieved. However, various output end styles tend to adopt different problem-solving tactics and impact the design of neural network models. Therefore, in the field of intelligent NLSCG and relevant tasks, we summarize four mainstream types from selected studies in terms of output end.

Semantic Parsing (SP): Semantic parsing is a classical school of computational linguistics and has always been an active research area. It maps natural language utterances into a semantic representation [76]. In the last few years, researchers have been trying hard to generate semantic representations such as regular expressions, if-then recipes, lambda calculus with their semantics, etc. The expressions generated by semantic parsing are associated with simple logic generally. The fuzzy mapping between each utterance and its logical form makes it more challenging. The relative entropy symmetry between the expressions and natural language gives more opportunities to produce a perfect semantic parser. However, researchers are encountering difficulties when dealing with complicated symbolic expressions [10].

Domain Specific Language Generation (DSLG): Generating types under the constraints of inherent modes usually take DSL as a medium. Currently, SQL generation is the most accessible direction, and this type of generation is limited to some meaningful fields under the DSL framework. On the other hand, DSL generation can be considered as the substage of the program synthesis process. By customizing the DSL suitable for the current generation target and context, the stage of DSL generation can be regarded as a buffer from ambiguous natural language to the high-level patterns in the program. DSLG is capable of assisting in program synthesis by bridging the gap.

Code Snippet Generation (CSG): Code snippet generation focuses on producing a small part of programs, which refers to fragments without advanced business logic flow in a project-level perspective. Researchers have tried to generate general-purpose languages like Python and Java, and are also starting to deliver impressive results. Compared with semantic parsing, the generation of this kind of code (1) usually involves a relatively large code scale and long dependency chain; and (2) encounters more complex generation scenarios. What is more, source code snippets are tightly constrained by syntax rules and affected by context. Moreover, numerous expressions and statements increase the difficulty of generation.

Program Synthesis (PS): Program generation is the ultimate goal and the thorny branch in the field of intelligent code generation. The asymmetries of information entropy between the program and natural language aggravate the difficulties of program synthesis. The mixture of the high-level patterns in the program and low-level detail implementations makes it extremely challenging to generate a one-shot result based on natural language. Therefore, a two-stage method was proposed to solve such problems [7,11].

\subsection{Source Code Generation Relevant Datasets}

There are multiple existing cross-domain datasets coupling with intelligent code generation tasks. To inspire in-depth research about this direction in readers, we combed all datasets appearing in selected studies. We created a profiling table for all selected studies, and purposely extracted and gathered information filled into the corresponding grid. We collected 21 attributes of different granularities from each study, including the adopted datasets. By integrating and removing the duplicate datasets, finally we obtain all the adopted datasets (36 in total). Due to the differences between the target language's complexity, a wide variation exists in intelligent generation model design, including the algorithm, the intermediate representation, and critical processing procedures. Therefore, these datasets and their variants are applied to different kinds of SCG tasks. Based on the characteristics and application fields of the datasets, we launched a finer-grained 
classification. Many classic datasets among them have been applied in semantic parsing tasks for years. Meanwhile, there are also emerging datasets created for current intelligent NLSCG. Aiming at the code generation task and according to the output end language complexity, the current aggregated datasets can be divided into six categories of four task types, as shown in Table 1 . The functional programming language can be conducted to a new category in terms of trends, and the proposed research has mentioned this drift.

Table 1. Output-oriented Dataset Classification.

\begin{tabular}{|c|c|c|c|}
\hline Classification & Generation Object & Dataset Name & Identifier \\
\hline \multirow{7}{*}{ Simple Logical Actions } & IFTTT & IFTTT 2015 & 1.1 \\
\hline & & IFTTT 2016 & 1.2 \\
\hline & \multirow{5}{*}{ Custom Actions } & SAIL & 1.3 \\
\hline & & SCENE & 1.4 \\
\hline & & ALCHEMY & 1.5 \\
\hline & & TANGRAMS & 1.6 \\
\hline & & Karel & 1.7 \\
\hline \multirow{11}{*}{ Logical Language } & Regular Expression & NL-RX & 2.1 \\
\hline & Prolog & JOBS & 2.2 \\
\hline & \multirow{5}{*}{ Lambda Calculus } & GEO & 2.3 \\
\hline & & ATIS & 2.4 \\
\hline & & NLMAPS & 2.5 \\
\hline & & SPADES & 2.6 \\
\hline & & GeoGrAnno & 2.7 \\
\hline & \multirow{4}{*}{ Lambda DCS } & WEBQUESTIONS & 2.8 \\
\hline & & OverNight & 2.9 \\
\hline & & WikiTableQuestions & 2.10 \\
\hline & & WEBQUESTIONSSP & 2.11 \\
\hline $\begin{array}{l}\text { Customized Domain } \\
\text { Specific Language }\end{array}$ & LISP-inspired DSL & ALGOLISP & 3.1 \\
\hline \multirow{12}{*}{$\begin{array}{c}\text { General-Purpose } \\
\text { Domain Specific Language }\end{array}$} & \multirow{2}{*}{ Graph Query } & GRAPHQUESTIONS & 4.1 \\
\hline & & ComplexWebQuestions & 4.2 \\
\hline & \multirow{9}{*}{ SQL } & GEO & 4.3 \\
\hline & & ATIS & 4.4 \\
\hline & & WikiSQL & 4.5 \\
\hline & & SENLIDB & 4.6 \\
\hline & & Scholar & 4.7 \\
\hline & & Textbook & 4.8 \\
\hline & & Advising & 4.9 \\
\hline & & Spider & 4.10 \\
\hline & & MIMICSQL & 4.11 \\
\hline & Bash & NL-Bash & 4.12 \\
\hline \multirow{4}{*}{$\begin{array}{l}\text { Advanced Programming } \\
\text { Language }\end{array}$} & \multirow{2}{*}{ Python } & DJANGO & 5.1 \\
\hline & & HS & 5.2 \\
\hline & \multirow{2}{*}{ Java } & MTG & 5.3 \\
\hline & & ACBJ & 5.4 \\
\hline \multirow{3}{*}{ Program } & API Usage & AML & 6.1 \\
\hline & \multirow{2}{*}{ Programmatic Context } & CONALA & 6.2 \\
\hline & & CONCODE & 6.3 \\
\hline
\end{tabular}


In particular, as a related field of code generation, semantic parsing aims to convert natural language utterances into logical forms with formal language. From our perspective, based on the complexity and abstraction of the formal language, we manually divide it into the simple logical actions and logical language. The simple logical actions belong to the most straightforward semantic parsing tasks. Some datasets possess a set of properties and actions that can be triggered by a pre-designed process, while some datasets attempt to describe the process control information (such as IFTTT) from a zones perspective. On the other hand, the logical language related datasets consist of natural language queries paired with a logical representation of their denotations. It has always been the most challenging branch task in the process of the semantic parsing field. There are various logical languages shown in datasets. The regular expression usually defines a pattern that verifies the feature of strings and has been widely used in various programming languages. As a more complex logical language initially employed to formal systems, lambda calculus applies a function to an argument and forms functions by abstraction [78] Prolog can be converted to equivalent statements in the lambda calculus, where some of the datasets are annotated with Prolog-style semantics too. Further, lambda dependency-based compositional semantics ( $\lambda$ DCS) was proposed in 2013; it is an alternate formal language that can be notationally simpler than lambda calculus [79]. Many semantic parsing tasks have been based on $\lambda$ DCS in recent years.

Domain-Specific Language (DSL) is very popular in the industry; it is a language designed for specific fields. The diversity between natural language and programs, which refers to the ambiguity of natural language, corresponds to the strictness of the program. Therefore, DSLG acts as a compromise to cope with this challenge, and the LISP-inspired DSL was designed for code generation instead of an existing programming language. It acts as a mediator to bridge the differences between natural language and source code. In addition, general-purpose DSLs like SQL and Bash are limited to the context of a specific platform or application and have been maturely applied in various development environments. Hence, the end-to end generation of DSL is becoming more accessible for recent SCG tasks.

The open-sourcing of the datasets promotes the automatic generation of source code snippets, especially the Trading Card Games (TCGs) like MTG and HS. TCGs map semistructured information, including textual description and categorical numerical attributes, to general-purpose programming languages like Java and Python. Some new datasets focus on code snippet generation which is constrained by the programming framework and cross-domain features. These burgeoning datasets enrich code snippet generation in real scenarios instead of being limited to one mode. Moreover, some datasets are collected for program synthesis tasks concentrating on code generation under the programmatic contexts (CONALA) or the essence of API usage (AML) in general-purpose languages.

Appendix A shows the details of all the summed-up datasets. These datasets employ the input in natural language or other semi-structured methods and are subsequently converted into various languages. Some datasets contain additional contextual information that constrains the generated source code, such as the generated SQL having to be compatible with the database schema, and the addition of context information would impact the entire model design.

\section{Insights Gained from NLSCG Research Backlog}

With the continuous improvement of deep learning and NLP technologies, researchers have attempted to model NLSCG from new perspectives. Researchers tend to stand on the shoulders of giants and get used to enhancing models based on the previous classic approaches. Exploring their initial research concerns is critical, and helps us follow up with improving the direction of this field. Therefore, we extract the research concerns from the selected studies and abstract them into the NLSCG research backlog, expecting to bring a more glittering view to future research. As shown in Figure 6, four disciplines in the treemap specify the critical research tasks for attempts and possible improvement directions, 
while the three tiers indicate different levels of details. The amount of studies related to a given backlog item determines the corresponding area's shade and size. We accumulate the research backlog in terms of resource, environment, boundary, enabler, utilities, etc., from the selected studies collected by the snowballing process. From the perspective of inherited research focuses and adaptive challenges, we summarize a taxonomy based on these research backlog items, which categorize all backlog items into four significant disciplines. (1) Language characteristics focuses on meeting challenges created by the inherent characteristics of natural language (NL) and source code (SC). (2) Target concerns emphasize the external features that are encountered during the generation process and that are able to be satisfied under a specific context or scenario. (3) Training refers to the difficulties confronted throughout the training process. (4) Context concentrates on processing the contextual information that exists in the input, output, and the entire generation problem. Despite this, "taxonomy" shows its immaturity and fragility; the scattered research backlog items gather together to present an overall comprehensible research treemap. Finally, we gain insights from the backlog of NLSCG research and elaborate the details in conjunction with specific studies.

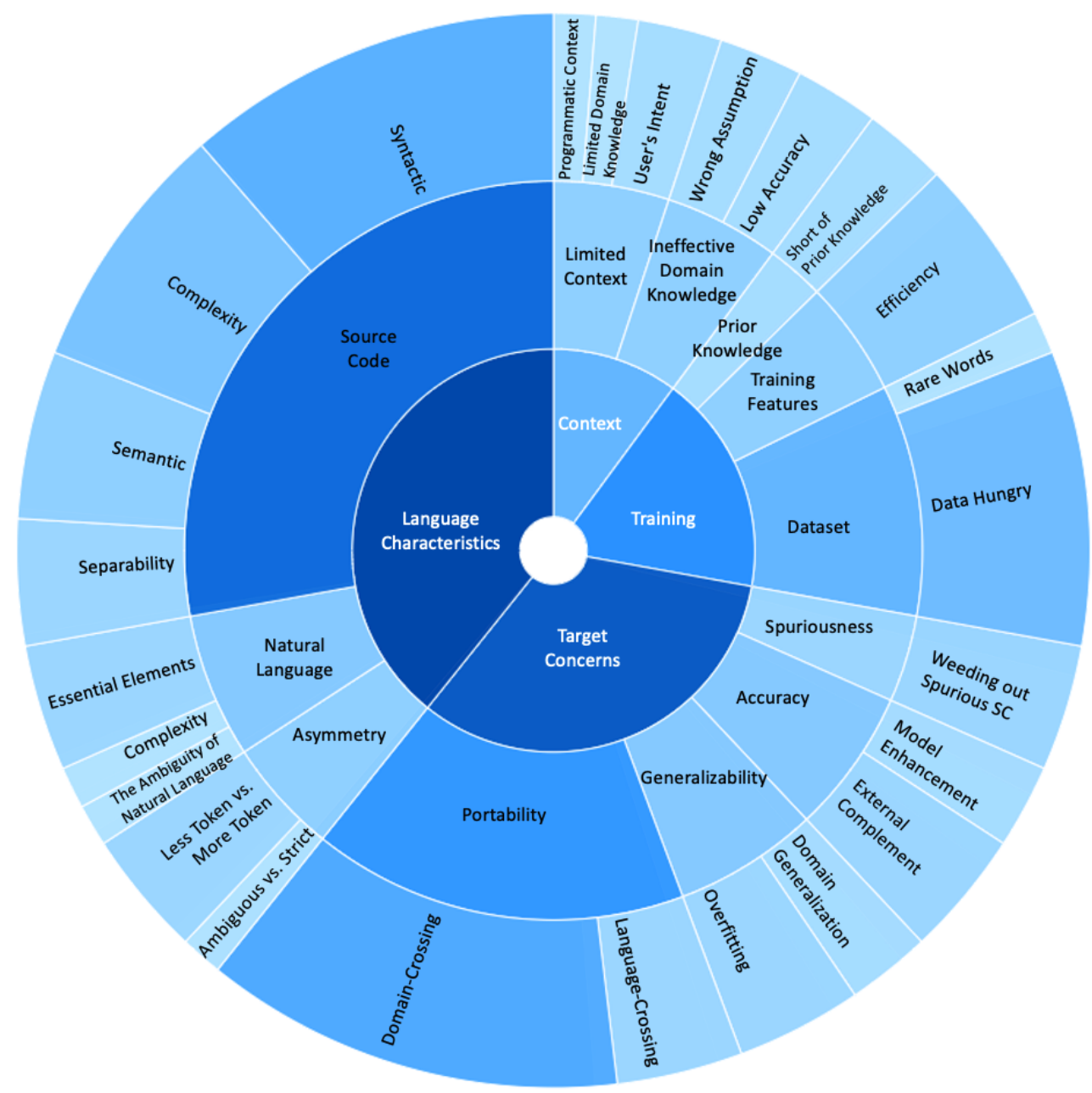

Figure 6. NLSCG Backlog Items.

\subsection{Language Characteristics}

Language is responsible for information transmission, and the mapping from NL to $\mathrm{SC}$ is one of the manifestations of abstract information transformation. NL and programming language possess various native characteristics that affect the model construction, and many targeted efforts have been put together to address such challenges. This subsec- 
tion describes the insights gained from language characteristic items according to the input and output language category, which can be refined into the basic appearance, contributory factors, expected impacts, attempted solutions, etc.

When modeling the SC end, distinct characteristics, including syntactic and semantic, and latent characteristics, including complexity and separability, accumulate to this part of backlog items. Syntactic dictates ensure well-formedness, type-sensitiveness, and executability, and pay close attention to semantic equivalence, program aliasing, and semantic consistency between the two ends in a semantic aspect. The complexity of the inherent structure in the SC end brings difficulties to sequential modeling. Obstacles include longdistance dependency problems, long decoding paths, the insufficient separation between high-level patterns and low-level implementations, etc. It is necessary to make trade-offs between tracking complete details and training efficiency. Research tasks on the NL end present as almost identical to the regular NLP field, which can be summarized from essential elements, complexity, and ambiguity. The adaption to the NLSCG tasks has put new requirements on the language ambiguity, owing to the asymmetries between the NL and SC ends. These asymmetries are not only reflected to a strict extent, but also in the number of tokens between the two ends.

\subsubsection{Source Code}

Syntactic. The generated SC must strictly satisfy a rich set of syntactic constraints, which can be easily extended to three aspects: Well-formedness, type-sensitiveness, and executability. To be specific, well-formedness can readily be kept by representing the SC as abstract syntax trees [4], while type-sensitiveness needs to guarantee the generated code is well typed. SC is often semantically brittle, and slight changes may drastically change the meaning of code [80], which may affect the executability. Besides, applying grammar-based decoding to general programming languages such as SQL is challenging as the AST may not sufficiently constrain its target [12]. Researchers devote themselves to tackle these fundamental problems through many different approaches, like formulating production rules [13], abstract syntax networks [4], program sketches [14], SQL template [15], targeted probability maximization [16], execution guidance [17], etc.

Complexity. The inherent structural information of SC induces it tricky-to-generate complex code correctly through the baseline approach. Take the case of RNN, which is considered as the primary fundamental network of the baseline approach. With RNN and its variants, memorizing large and complex structures is arduous [18], and capturing long sequences extracted from AST is inappropriate for RNN [19]. Besides, the long-distance dependency problem, occurring between code elements where their positions are far apart in space [20], exacerbates the unsuitability of RNN-based modeling. What is more, the process of converting SC into a flattened sequence through AST can easily lead to long decoding paths, even for short snippets of SC [21]. Some over-detailed expansions will not provide fancy information for constructing neural decoding models; instead, this will bring in interference and result in a meaningless waste of training resources. Complexity also affects the DSLG tasks, especially the generation of nested SQL queries [22], and we can simplify the complexity of the generator and improve performance by ignoring the order of fields and condition attributes [23].

Semantic. Prevalent challenges exist across diverse SC categories in the aspect of semantic and syntactic elements. Meanwhile, slight differences appear among these elements in complexity and significance. In terms of semantics, $\mathrm{Xu}$ [24] finds that different SQL queries may be equivalent to each other due to the commutativity and associativity, which lead to the futility of the sequence in certain SQL positions, like tokens in attributes in SELECT and WHERE clauses. However, the order of these constraints would affect the performance of a sequence-to-sequence-style model, which is considered as the baseline model in the SCG tasks. In addition, many different programs can frequently satisfy one given the NL specification, which suggests a phenomenon known as "program aliasing" [16,25], and this type of limitation pervades all SC categories. 
Separability. Program synthesis is perceived as the most challenging of the intelligent NLSCG tasks due to the insufficient separation between high-level patterns and lowlevel implementation details [7]. The low-level features, such as variable names and intermediate results, would impede the learning process. While the high-level patterns are challenging to learn from datasets, a combinatorial, syntax-guided program synthesizer can easily accomplish this task [81]. Therefore, researchers [14,26] have attempted to get the decomposition disentangled high-level features from low-level features, and enable decoders to model at different levels of granularity.

\subsubsection{Natural Language}

Essential Elements. On account of the two essential elements of natural language, vocabulary and syntax, tentative efforts have been made to get improvements. Some studies merely extract word order features depending on SeqLSTM while neglecting useful syntactic information [27]. To address the problem, authors [28] tried to employ syntactic trees (like CCG and CFG) instead of flat sequence on the input end to thoroughly capture NL information based on dependency parsing and constituency parsing . Meanwhile, it is noticeable that the characteristics of RNN structure make it difficult to learn proper representation from the diverse vocabulary and syntax [29].

Complexity. In particular scenarios, the input question composed of sub-questions might be too long to understand. Understanding each sub-question could contribute to the semantic parsing of the original complex question [30]. The decomposition of complex questions can be considered as the predecessor task of NLSCG to alleviate the difficulties of understanding. Rule-based methods have been proposed [82], but experts and massive design efforts have to be devoted to the task. A neural question decomposer has been tentatively proposed to identify sub-questions more accurately [30].

Ambiguity. Another problem that blocks the research process is language ambiguity [31]. After a long-term NL evolution, people tend to express their thoughts relatively concisely, such as with fewer words and shorter phrases. More importantly, ambiguous language can be accurately understood only on the premise that the whole context is comprehended. The linguistic ambiguity problems may affect the meaning of the syntactic elements [83], which aggravates the difficulty of generating the corresponding code.

\subsubsection{Asymmetries between Natural Language and Source Code}

Encoder-decoder architecture is widely applied to intelligent NLSCG and its similar tasks. The encoder takes NL as the input sequence, converts it into specific representations, and then transforms it into the desired code structures via the decoder. This section attempts to summarize the research backlog affected by the asymmetries between encoder and decoder.

Less Token vs. More Token. Many studies find that apparent asymmetries in the number of tokens between input and output end give rise to beset difficulties in building the generative models $[18,19,25]$. These asymmetries can easily attribute to the larger number of tokens in the source code end. To ensure source code can be modeled without missing rich structure information, converting source code into tokens depending on a specific traversal approach from AST is prevalent. Extracting tokens on account of the tree-based approach can easily lead to rapid quantity growth compared to the original source code, which exacerbates the appearance of asymmetry. CNN-based models [19], retrieval-based generation [18], and pre-training distributions of SC and NL [25] are proposed to address this backlog item.

Ambiguous vs. Strict. As an essential attribute of NL, ambiguity brings difficulties in describing the exact requirements clearly [9]. In contrast, the source code seems to be sophisticated equipment with precision and unequivocal representation. A slight difference would impact on syntax, executability, and even semantics of source code. Therefore, extra efforts must be devoted to coping with the transformation from ambiguous language to 
strict language. Research [9] tackled this problem by attaching input and output examples to synthesize programs.

\subsection{Target Concerns}

Concerns running through the entire modeling process are worthy of consideration, and many studies attempt to concentrate on it to get potential improvements. In this section, we summarize the four chief features that researchers devote more attention to. We inspect the portability and generalizability from a global perspective, and the accuracy and spuriousness focus on evaluating the generation task itself, where we discuss their derivation, usage scenario, and tentative solutions. The concerns expressed by these models can be applied to evaluate the external model learning ability to a certain extent. By studying the current limitations of these concerns, favorable directions to improve the model became apparent.

For achieving better performance, researchers restrict the model to a limited domain, resulting in domain-crossing problems. The new release of cross-domain datasets and attempts at learning methods have greatly alleviated this difficulty. Since the semantic equivalence between the two languages cannot be calculated, it is almost impossible to construct a cross-language generative model under the existing technical conditions. This backlog item still has a long way to grow. In terms of generalization ability, overfitting is a common problem, and recursive generative architecture and reinforcement learning methods have been presented to tackle this problem. External complement and model enhancement perspectives can improve the accuracy of the model. Dataset augmentation, search space optimization, dialog-based generation, and two-stage generation are prevalent approaches in external complement. Researchers are improving the model accuracy by discovering potential limitations in architecture strategies and learning approaches. Lastly, to solve spurious programs, the auxiliary reward function of reinforcement learning and the mechanism to eliminate spurious programs during the training process are practical approaches.

\subsubsection{Portability}

Domain crossing. Generating target code with high precision across domains has become the main bottleneck of NLSCG tasks tightly coupled with domain knowledge. As elaborated in Section 5.4.2, domain knowledge utilization shows progressive features, (1) investigating whether to utilize domain knowledge in the budding phase; (2) discussing how to utilize domain knowledge in the rising phase efficiently; and (3) addressing how to utilize domain knowledge sufficiently under the premise of crossing domains in the perfection phase. The inherent ambiguity exhibited by NL restricts the NLSCG task to specific application scenarios [32]. Depending on manually designed features, many previous works build models with a domain-specific specialty [33], and these models learn to match semantic parsing results rather than truly learn to understand the meanings of inputs [34], making it challenging to adapt to new domains. Building the model targeted on one specific domain (i.e., database, table) [35] or binding interactive guidance from users has become the primary approach that existing technologies try in order to achieve high accuracy. Hence, existing techniques for NL-based synthesizing of the corresponding code , SQL in particular, are not database agnostic (i.e., do not require database-specific training or customization) [36]. One reason is that the training datasets applied in the NLSCG tasks based on supervised learning are concentrated on the particular domain while ignoring data previously collected for other domains. The popular Overnight [84] dataset containing eight domains has greatly alleviated this problem. In addition to mainstream supervised learning, some general cross-domain learning categories have been introduced to NLSCG, including meta-learning [37] and transfer learning [38], and have achieved confident performance.

Language crossing. NLSCG tasks embrace various code types constrained by relatively unique grammatical rules. To achieve the general-purpose generation and ensure the 
syntactic well-formedness of the generated code, it is necessary to design a parser that explicitly reflects the domain-dependent grammar of code embedded in the model [39]. Prior work proposes a model that performs the task from text descriptions to regular expressions, utilizing a domain-specific component that computes the semantic equivalence between two regular expressions [85]. However, the effectiveness of this approach is extremely dependent on the component mentioned above, leading to trouble with generalizing to other languages since the semantic equivalence calculations are not possible across other formal languages [40]. Therefore, addressing this issue with general-purpose grammar models has become a considerable exploration direction.

\subsubsection{Generalizability}

Overfitting. Overfitting remembers superfluous minor features of the training data and reduces the generalization ability. A model with good generalization must be able to perform exceptionally well outside the training set. In terms of the NL end, due to the particularity of real-world NLSCG tasks, the large and varied vocabulary with many rare words naturally intensifies overfitting [41]. When it comes to the SC end, the more extraordinary complexity generation scenarios, like the multi-schema problem in SQL, would aggravate the occurrence of overfitting [42]. Recent work [10] has discovered that incorporating the notion of recursion into neural program synthesis architecture or leveraging reinforcement learning instead of supervised techniques could significantly improve model generalizability.

Domain Generalization. Solving the problems of limited training samples and data distribution conversion can improve the domain generalization problem to a certain extent. As described in Section 5.4.1, domain crossing can evaluate the portability of a model, which means training based on one domain and then smoothly applied to another one without any additional training data. However, it is challenging to achieve this goal as, in the training process, not all the target domain KB constants (relationships and entities) can be observed at the training stage [15], and emerging domains will break the pre-adapted models. Furthermore, mainstream complex SQL query generation methods often take SQL templates or slots as the intermediate absorber and impair the ability to generate code of unseen templates [43].

\subsubsection{Accuracy}

External Complement. Improvements based on the model itself are prone to touch the ceiling. From an engineering thinking perspective, complementing specific components externally, the model can more effectively improve the performance of the generated tasks. Inspired by successful application in machine translation, a retrieval-based approach has been introduced into neural code generation [18]. For instance, studies [6,44] gain an insight that the parser still maintains high recall when incorporating the n-best predictions into the gold-standard generated code most of the time. Thus, by reranking the n-best list of candidate code snippets, they investigate potential actions to improve the precision.

Model Enhancement. Popular external complements, such as training set enrichment [45], search space optimization $[6,18,46]$, purposeful supplementation of context information [32], two-stage generation $[7,14,36]$, etc., are prevalent approaches to improving the accuracy of NLSCG tasks. However, some researchers are dedicated to focusing on a pure end-toend approach. They learn experiences from historical success models, discovering latent limitations from the perspectives of model architecture strategy and learning approach, and improve precision within an accomplishable range. Research [8] has proposed a bare neural model, without any search, additional postprocessing, or other forms of auxiliary means, and still achieved state-of-the-art test-set accuracy. Instead of designing multiple decoders from different language components or adding extra controllers for expansion of production rules, authors [47] have proposed a fine-grain control over the decoding process while retaining the simplicity of the sequence structure. 


\subsubsection{Spuriousness}

Weeding out Spurious SC. NLSCG sometimes encountered spurious programs, which are prevalent in any general-purpose programming language. Spurious programs denote that the incorrect utterances accidentally produce correct results yet do not reflect the actual semantics. Research [48-50] in selected studies explored this topic. Spurious programs would provide wrong signals for the generation model, developing in an increasingly biased direction. This can be alleviated by using the auxiliary reward function derived from reinforcement learning [48] or designing a mechanism to eliminate spurious programs during training [50].

\subsection{Training}

Sometimes the failure of training neural networks occurs in silence; Karpathy [86] has proposed a recipe to achieve better performance for training neural networks. Compared with adopting inertial and experience-based tricks with a kind of elusiveness, researchers are trying to find the breakthrough from visible limitations, including training dataset and training characteristics, to get improvements. In this subsection, we summarize the research backlog items related to training that researchers discover in the NLSCG field to inspire potential enhancements.

Data-hungry and rare word problems are widespread in machine learning training tasks. The former can be alleviated via semi/unsupervised learning, data augmentation, and releasing new datasets to a certain degree. In dealing with rare words problem, the only thing to pay attention to is how to accurately embrace the successful experience applied in other fields to the distinctive SC end. Capturing all tiny details on the SC end would inevitably lead to computational challenges, and researchers should make trade-offs between complicated deep learning models and training efficiency. In addition, injecting prior knowledge would improve model performance, and successful attempts are based on the data recombination process.

\subsubsection{Dataset}

Data Hungry. The dataset is one of the essential factors determining the upper bound of the model performance to some extent. The abundance of high-quality labeled data is critical for effectively training supervised models [31,51]. However, manually annotating NL utterances with their corresponding SC is expensive, cumbersome, and time consuming $[10,38,52,53]$. While emerging datasets have sprung up, due to the limitations in quantity, quality, and domain-crossing, the limited availability of labeled data is still becoming the primary bottleneck for data-driven supervised models [49].

Rare Words. It is challenging to learn representations of rare words with a neural language model. The entire word sequence containing rare words would be underestimated due to the influence of rare words on the context of neighboring words [87]. Words that are rare in the dataset are unable to learn good enough parameters [88]. The hybrid method combining significant example retrieval and neural models has been proven to be successful in processing rare words [89]. Hayati et al. [18] deal with rare words through constructing n-gram over subtrees to exploit the code structural similarity.

\subsubsection{Training Features}

Efficiency. Modeling source code with large formal grammars can easily lead to long decoding paths, even for short code snippets [21]. The training of deep neural networks with complex topologies is difficult, time consuming, and error-prone [54]. Researchers should find a way to make a trade-off between complicated deep learning models and training efficiency. Zhong et al. [55] discovered that the output space of the softmax in the Seq2Seq model would be unnecessarily large if the generated sequence cannot be restricted to a particular list. Computational challenges occur concerning supervised learning, as well as concerning semi-supervised learning. A semi-supervised autoencoder tends to convert the transformation object from $(\mathrm{x} \rightarrow \mathrm{y})$ to $(\mathrm{y} \rightarrow \mathrm{x} \rightarrow \mathrm{y})$ by considering the input 
sequence as a latent variable, where $\mathrm{x}$ is the input sequence, and $\mathrm{y}$ represents the output sequence. However, modeling the latent variable as a series of discrete symbols drawn from multinomial distributions will bring serious computational challenges, as it requires marginalizing over the space of latent sequences $\sum_{x}^{*}[51]$.

\subsubsection{Prior Knowledge}

Lack of Prior Knowledge. Recently, some NLSCG tasks tried to build more flexible models and generate the corresponding code via learning more general grammars and features while trimming lots of task-specific prior knowledge. Compared with standard semantic parsers, this modeling flexibility is attributed to the common application of minimal feature engineering represented by RNNs. Such knowledge plays a critical role in understanding modeling limitations, and the parser would get better performance under the constraints of task-specific prior knowledge [56]. Jia and Liang [57] have proposed a data recombination framework which induces a generative model from the training data and samples from it to generate new training examples with available prior knowledge.

\subsection{Context}

The context indicates information about the circumstances in which something or someone is located, which can influence and assist users in understanding the original intention to a certain extent. In the entire process of NLSCG, three components would be affected by the context, including the input end, the output end, and the relatively special domain knowledge across the whole generation problem (especially for instances in DSLG and SP). The lack and insufficient application of such information impel us to discover these research backlog items.

However, rare existing works make sufficient use of the context information, which accumulates the backlog items of ineffective domain knowledge. The inefficiency is mainly reflected in the low accuracy caused by the lack of accurate knowledge embedding and the preconceived wrong assumptions before model design. Besides, the limited context indicates the restricted context from the user's intents and programmatic and generation problem perspectives. The present items mainly lie in the narrow scope of the context, insufficient utilization manner, and lack of reasonable solutions, etc.

\subsubsection{Ineffective Domain Knowledge}

Low Accuracy. The input NL query often contains keywords that can be specific to the underlying database. Shreds of evidence show that those words are crucial to many downstream actions, including inferring column names and condition values in the SQL query [58]. Some particular tasks of NLSCG constrained by additional domain knowledge to generate proper SC present significant differences in taking advantage of domain knowledge. Research [59] notices that some studies do not utilize domain knowledge; specifically, those missing or lacking accurate embeddings exist between the database schema entity and the input query.

Wrong Assumption. Researchers $[43,58]$ find many models assume that users have an exceptionally familiar sense of the domain knowledge, such as knowing about the exact column names and string entries in that table. They copy the question words from the input query directly via a soft-alignment mechanism. However, the mismatch between question words and column names (or cells) exacerbates the incorrectness and non-executability of the generated results. Under these circumstances, even if the embeddings of domain knowledge reach perfection, the performance of downstream tasks could not meet the expectations, and it seems to be counterproductive occasionally.

\subsubsection{Limited Context}

User's Intent. Acquiring users' real intentions follows the principle of gradual improvement. However, the reality is that existing works tend to assume that the corresponding source code can be generated end to end from one single NL description [60]. 
Therefore, many users' fundamental details are omitted, yet the generator does not have the capabilities to figure out the user's real intentions via their initial description.

Programmatic Context. The mainstream generating approaches of NLSCG have restricted the relevant code to a limited language and code environment. In most cases, the generated code focuses on more straightforward scenarios and context, so that very few of them take into account programmatic context. For example, the fixed code templates generate only parts of a method with a predefined structure, such as IFTTT and custom actions; a fixed context generates the body of the same method within a single fixed class, such as code snippets; or no context at all generates code tokens from the flat $\mathrm{SC}$ sequences separately, such as SQL slots [61]. All those mentioned above are still a long way from the executable program. While these fragments have motivated NLSCG solutions in model design and architecture selection, the generation scenario with the programmatic context is more complicated and presents its practicality.

Simple Context. In NLSCG tasks, mapping NL utterance to the target SC under the context of special domain knowledge, such as generating SQL constrained by database schema, semantic parsing associated with its own knowledge base, is a particular instance. The number of domains, schemas, and tables, along with users' intentions, can determine the difficulty level of SQL generation tasks. Many datasets about one domain contain only one database schema for that domain; more seriously, some schema only contain one table [42]. Consequently, many existing works are often formed into simple and crude applications, as complex scenarios like multi-table cascade queries by foreign key can never happen concerning these works.

\section{Perspectives on NLSCG Latest Technology Landscape}

This section summarizes the foremost important part of the NLSCG models through an intensive investigation of the selected studies. The generative model can be regarded as a particular data processing problem, with which it is easy to depict the entire generation process clearly by utilizing a pipeline-based approach. Based on the two main processing pipelines of NLSCG, namely the NL end (description of requirements and intents) and the SC end (executable source code in a specific context); regarding research backlog as guidelines, we decompose raw information from selected studies, and convert them into essential components, and then build interactions according to the data processing chain and the conventional deep learning model pipeline. We propose a latest NLSCG technology landscape illustrated with a metro map metaphor, to explain the NLSCG utilities as vividly as possible. On this basis, the leading technical tactics adopted by each component are elaborated gradually to stimulate more possibilities for model construction.

Regarding NLSCG tasks as data processing problems, we use the pipeline-based diagram to produce a thorough elaboration of the current utilities shown in Figure 7. Like two ends of a scale, we could go through two main routes covering the generation process from NL to SC when traveling along with pipelines in the landscape diagram. Besides, another 11 routes run through the main routes directly or indirectly with the identification of corresponding color and guideboard. During the crossroad, important components are highlighted in the form of different types of shapes. The critical technical points are displayed in the diagram in the form of a circle point. The solid path indicates that information can be directly transmitted between the two main lines, while the dashed path cannot. Table 2 describes the significant routes and intersections deriving from the landscape. Here we consider them as the actuating routes and enabling factors that motivate the further improvements of the NLSCG field. The representative studies which indeed describe the corresponding enabling factors are also exhibited in this table.

NLSCG maps natural language (NL) utterances into an executable representation. As mentioned in Section 4.3, these executable representations refer to logical actions, logical language, customized DSL, general-purpose DSL, advanced programming language, and program. There are some scenarios where the NLSCG process depends on a specific programmatic context [61]. The input representation is typically executed against a problem 
context, such as a knowledge base [62] or database [58], to generate the desired output. For the problem context that plays an essential role in semantic analysis and domain-specific language generation tasks, the context scope, the context-embedding approaches, and the manners of combining context with the NL end are worthy of attention.

Different from the ambiguity of natural language, the source code is constrained by an underlying syntax or grammar. Such constraints can usually be converted into an intermediate structure with AST as the typical grammar criterion. The complexity of the intermediate tree structure brings difficulties in leveraging information with rich structures, which attracts most researchers' attention. The prerequisite for taking full advantage of such information is to clarify the structural embedding basis before gaining representations, which is different from the conventional word or character level granularity of the NL. The inherent structural information in the SC constrained by the syntax rules has shown its utilization characteristics of diversity; different state propagation directions, grammar conversion criterion, distinguishing basis, and the random combinations between them have a profound impact on the design of subsequent embedding algorithms. Considering the complete embedding for both ends, researchers take sequence or structure as the modeling object to construct the network. Researchers also make trade-offs between the levels of details and the final network complexity in the structure-embedding process to ensure the training efficiency of the model.

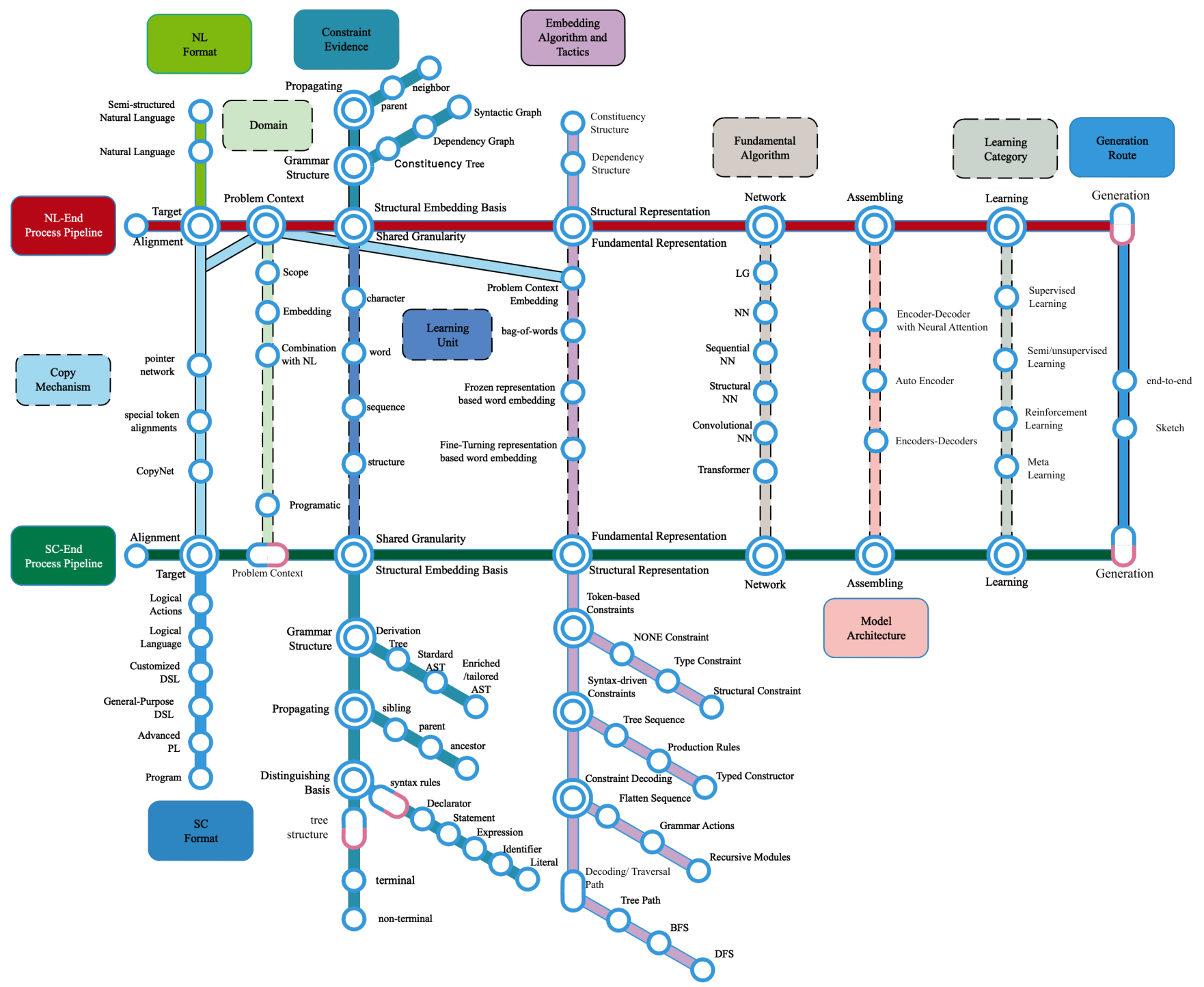

Figure 7. NLSCG Technology Landscape. 
Table 2. Significant Enabling Factors in the Landscape.

\begin{tabular}{|c|c|c|c|}
\hline $\begin{array}{l}\text { Actuating } \\
\text { Routes }\end{array}$ & $\begin{array}{l}\text { Enabling } \\
\text { Factors }\end{array}$ & Description & $\begin{array}{l}\text { Representative } \\
\text { Studies }\end{array}$ \\
\hline $\begin{array}{l}\text { Copy } \\
\text { Mechanism }\end{array}$ & Alignment & $\begin{array}{l}\text { Outline the most classic and widespread strategies that align } \\
\text { the NL end and SC end. }\end{array}$ & S2,S3,S6,etc. \\
\hline Domain & $\begin{array}{l}\text { Problem } \\
\text { Context }\end{array}$ & $\begin{array}{l}\text { Focus on the critical contextual information and how it } \\
\text { assimilates into the generative model. }\end{array}$ & $\begin{array}{l}\mathrm{S} 7, \mathrm{~S} 34, \mathrm{~S} 35, \mathrm{~S} 45, \\
\mathrm{~S} 56, \mathrm{~S} 63, \mathrm{~S} 65, \text { etc. }\end{array}$ \\
\hline $\begin{array}{l}\text { Learning } \\
\text { Unit }\end{array}$ & $\begin{array}{l}\text { Shared } \\
\text { Granularity }\end{array}$ & $\begin{array}{l}\text { Sketch the granularity of the participants' vectorization unit } \\
\text { in the encoding stage (word or character) and modeling object } \\
\text { in the network construction stage (sequence or structure). }\end{array}$ & S1,S22,etc. \\
\hline $\begin{array}{l}\text { Constraint } \\
\text { Evidence }\end{array}$ & $\begin{array}{l}\text { Structural } \\
\text { Embedding } \\
\text { Basis }\end{array}$ & $\begin{array}{l}\text { Discuss the basis for achieving structural embedding from } \\
\text { different views. }\end{array}$ & $\begin{array}{l}\text { S2,S3,S14,S36, } \\
\text { S50,S58,S64,etc. }\end{array}$ \\
\hline $\begin{array}{l}\text { Constraint } \\
\text { Evidence }\end{array}$ & $\begin{array}{l}\text { Grammar } \\
\text { Structure }\end{array}$ & $\begin{array}{l}\text { Describe the extraction criteria for getting intermediate } \\
\text { structure. }\end{array}$ & S36,S58,S64,etc. \\
\hline $\begin{array}{l}\text { Constraint } \\
\text { Evidence }\end{array}$ & Propagation & $\begin{array}{l}\text { Discuss the additional state propagation between nodes at } \\
\text { the different relative position in the grammar structure. }\end{array}$ & S6,S9,S21, etc. \\
\hline $\begin{array}{l}\text { Constraint } \\
\text { Evidence }\end{array}$ & $\begin{array}{l}\text { Distinguishing } \\
\text { Basis }\end{array}$ & $\begin{array}{l}\text { Indicate the distinguishing basis of different nodes in the } \\
\text { grammar structure in the subsequent modeling process. }\end{array}$ & S2,S3,etc. \\
\hline $\begin{array}{l}\text { Embedding } \\
\text { Algorithm } \\
\text { and Tactics }\end{array}$ & $\begin{array}{l}\text { Fundamental } \\
\text { Representation }\end{array}$ & $\begin{array}{l}\text { Investigate the conventional embedding algorithms and } \\
\text { tactics based on the original sequence input. }\end{array}$ & $\mathrm{S} 1, \mathrm{~S} 8, \mathrm{~S} 15, \mathrm{~S} 30$,etc. \\
\hline $\begin{array}{l}\text { Embedding } \\
\text { Algorithm } \\
\text { and Tactics }\end{array}$ & $\begin{array}{l}\text { Structural } \\
\text { Representation }\end{array}$ & $\begin{array}{l}\text { Explore the common embedding algorithms and tactics based } \\
\text { on the intermediate structure. }\end{array}$ & $\begin{array}{l}\text { S11,S14,S15,S50, } \\
\text { S66,etc. }\end{array}$ \\
\hline $\begin{array}{l}\text { Embedding } \\
\text { Algorithm } \\
\text { and Tactics }\end{array}$ & $\begin{array}{l}\text { Token-based } \\
\text { Constraints }\end{array}$ & $\begin{array}{l}\text { Summarize the embedding strategies that consider tokens as } \\
\text { the decoding object and hold the extended constraint during } \\
\text { the decoding process. }\end{array}$ & $\begin{array}{l}\text { S5,S7,S16,S20, } \\
\text { S26,S34,etc. }\end{array}$ \\
\hline $\begin{array}{l}\text { Embedding } \\
\text { Algorithm } \\
\text { and Tactics }\end{array}$ & $\begin{array}{l}\text { Syntax-driven } \\
\text { Constraints }\end{array}$ & $\begin{array}{l}\text { Summarize the embedding strategies that utilize intermediate } \\
\text { structure as the springboard, granularity and syntactic } \\
\text { constraints as the foundation and standard, and they work } \\
\text { together to extract the structure information. }\end{array}$ & $\begin{array}{l}\text { S2,S3,S11,S15, } \\
\text { S25,S44,S45,etc. }\end{array}$ \\
\hline $\begin{array}{l}\text { Embedding } \\
\text { Algorithm } \\
\text { and Tactics }\end{array}$ & $\begin{array}{l}\text { Constraint } \\
\text { Decoding }\end{array}$ & $\begin{array}{l}\text { Summarize on the strategy for attaching critical constraints to } \\
\text { the decoding inference stage to ensure the syntactic correctness } \\
\text { and semantic accuracy of the generated result. }\end{array}$ & $\begin{array}{l}\mathrm{S} 2, \mathrm{~S} 3, \mathrm{~S} 8, \mathrm{~S} 10, \\
\mathrm{~S} 11, \mathrm{~S} 15, \text { etc. }\end{array}$ \\
\hline $\begin{array}{l}\text { Fundamental } \\
\text { Algorithm }\end{array}$ & Network & $\begin{array}{l}\text { Introduce the widely adopted networks and variants and } \\
\text { the approaches to integrating the learning unit, constraint } \\
\text { evidence, and embedding algorithm and tactics. }\end{array}$ & $\begin{array}{l}\text { S1,S7,S11,S14, } \\
\text { S22,S23,S52,etc. }\end{array}$ \\
\hline $\begin{array}{l}\text { Model } \\
\text { Architecture }\end{array}$ & Assembling & $\begin{array}{l}\text { Outline the assembling paradigms that assemble multiple } \\
\text { encoders or decoders composed of fundamental networks. }\end{array}$ & $\begin{array}{l}\text { S8,S11,S15,S23, } \\
\text { S51,S63,etc. }\end{array}$ \\
\hline $\begin{array}{l}\text { Learning } \\
\text { Category }\end{array}$ & Learning & $\begin{array}{l}\text { Summarize the learning categories that NLSCG attempts and } \\
\text { their adaptive scenario and superiorities. }\end{array}$ & $\begin{array}{l}\text { S8,S15,S16,S24, } \\
\text { S28,S30,S55,etc. }\end{array}$ \\
\hline $\begin{array}{l}\text { Generation } \\
\text { Route }\end{array}$ & Generation & $\begin{array}{l}\text { Discuss the generation manner in the way of end-to end } \\
\text { or via an intermediate stage. }\end{array}$ & $\mathrm{S} 6, \mathrm{~S} 34, \mathrm{~S} 35, \mathrm{~S} 41$,etc \\
\hline
\end{tabular}

After confirming the granularity of the learning starting point, the relevant NL should be encoded into the representations that the computer can recognize, with the expectation of not losing information during the encoding process. At this stage, comparing NL and SC, current explorations present a relatively different situation and tendency. Overall, various embedding algorithms are applied to the NL end, while few researches have focused merely on the bare source code encoding. The majority of the NL end and minority of the SC end are regarded as flat sequences, adopting representation learning algorithms commonly applied in the NLP field. In contrast, both ends attempt different capture strategies for extracting information via the structure modeling object. By employing the 
syntactic analysis thinking, the NL end directly builds tree-based neural networks on the intermediate syntactic structure. The representation of structural information from the SC end shows its own particularity, with the granularity and structural embedding basis as the cornerstones. These foundations serve as inspirations to design constraint strategy and decoding process, and gradually develop into mature approaches. Current studies have made efforts in two types of constraints, namely token-based constraints and syntax-driven constraints; combining specific constraints with the appropriate decoding process would unleash huge creative potentials. These constraints and decoding processes establish a benchmark for accurately tracking structural information. Further, various methods are branched on this mainline to perform subsequent modeling and deriving to different factions.

To ensure the syntactic correctness and semantic accuracy of the generated SC, the constraint decoding attaches critical structural constraints to the decoding inference stage. Afterward, a distribution is produced over these SC by modeling and ultimately screening high scoring results after the searching procedure under this model. Similar to familiar intelligent generation tasks, the fundamental algorithms adopted by NLSCG are based on popular deep learning models and their variants. These actualities hold for both NL and SC end modeling. Multiple types of comprehensive networks, including sequential NN (RNN, LSTM, etc.), convolutional NN (CNN), and structural NN (tree NN and graph NN), are attempted in this field, and the emerging modeling algorithms like Transformer are applied to NLSCG and have gradually shown their superiority.

In most cases, the complexities of NLSCG tasks result in the infeasibility of generation relying on one single neural network. Therefore, the encoder-decoder framework with neural attention has become the baseline architecture of NLSCG, and the assembly of various models on this basis is also becoming increasingly mainstream. The attention mechanism was initially introduced to align the information between NL end and SC end, and it is frequently employed in the splicing and integration process of the network.

The learning algorithm is employed to update the model's parameters on account of the training dataset. Supervised learning still occupies the mainstream category with its more accessible training and higher accuracy. Semi/unsupervised learning has also been introduced as the auxiliary method to solve the data-hungry problem. Reinforcement learning has also been introduced to enhance performance and generalization. In addition, metalearning is constantly being introduced into this area to deal with cross-domain problems.

Hybrid methods have proven to be effective in NLSCG tasks. Hence, we could transfer between different routes from the starting, terminal, and intermediate points in the landscape to inspire potential improvements of NLSCG utilities. The end-to-end generation strategy sustained by technologies and components linked by the pipeline still occupies the mainstream position of the current generation task's technology stack. However, many studies shed a different light on external complementary methods, with the hope of making the next leap in performance and domain-crossing. Genres based on search concepts, including combining retrieval methods into neural code generation models [18,46], reranking a list of potential candidates [6], etc., are relatively feasible. Besides, data augmentation $[45,63]$ processing based on training datasets, as well as the two-stage [52] and sketch [24]-based generation tactics, are also worthy of consideration.

\section{Conclusions and Future Directions}

SCG has been studied for a long time, and the NLSCG shows its popularity with the increasing adaptation maturity of deep learning techniques. This survey provides insights for NLSCG from laborious and deliberate exploration of 66 relevant studies collected by the well-designed snowballing process. We extract preliminary information from the selected studies, review the overall development trend in recent years, summarize the frequently used dataset, and refine the types of NLSCG research tasks, consequently promoting the understanding of the NLSCG problem. Then the research backlog is filled by considering critical motivations and priorities under the guidance of the research journey 
map. We extract a taxonomy based on the research backlog items through a circular treemap regarding the inherited research focuses and adaptive challenges. Then we summarize the insights gained from the NLSCG research backlog and elaborate the details. Finally, based on the two main processing pipelines of NLSCG, we propose a technology landscape of NLSCG to explain the NLSCG utilities as vividly as possible. The technology landscape depicts the critical fundamental components, correlations between components, and leading technical tactics, stimulating more possibilities for model construction.

We agree that the following issues need to be addressed in future work. First of all, there is no precise horizontal comparison between the selected studies. The reasons are as follows: (1) There are significant differences in various types of generation tasks, which hinder the comparison; (2) most selected studies use different datasets and evaluation benchmarks, which increase the difficulty of comparison. Secondly, this article only introduces main components in the NLSCG landscape due to space limitations, while ignoring important details about each technical solution, which need to be elaborated in future research.

Author Contributions: Conceptualization, Y.L. and C.Y. (Chen Yang); methodology, Y.L. and C.Y. (Chen Yang); formal analysis, C.Y. (Chen Yang); investigation, C.Y. (Chen Yang); resources, C.Y. (Chen Yang); writing — original draft preparation, C.Y. (Chen Yang); writing—review and editing, Y.L. and C.Y. (Chen Yang); supervision, Y.L. and C.Y. (Changqing Yin); project administration, Y.L.; funding acquisition, Y.L. All authors have read and agreed to the published version of the manuscript.

Funding: This research was funded by the National Key Research and Development Program of China under grand number 2018YFC0704600.

Institutional Review Board Statement: Not applicable.

Informed Consent Statement: Not applicable.

Data Availability Statement: Selected Studies List Ref: https://github.com/Ada12/NLSCG Research/blob/main/selected-studies-list.md, accessed on 20 August 2021.

Conflicts of Interest: The authors declare no conflict of interest.

\author{
Abbreviations \\ The following abbreviations are used in this manuscript: \\ NLSCG Natural Language based Source Code Generation \\ SCG Source Code Generation \\ NL Natural Language \\ SC Source Code \\ EMA Enriched Models based Approach \\ DSLA Domain-Specific Language based Approach \\ FLA Formal Language based Approach \\ GMA Graphic Modeling based Approach \\ NRA Nature Requirement based Approach \\ SP Semantic Parsing \\ DSLG Domain Specific Language Generation \\ CSG Code Snippet Generation \\ PS Program Synthesis \\ TCGs Trading Card Games
}




\section{Appendix A. Dataset List}

Table A1. Datasets.

\begin{tabular}{|c|c|c|c|c|c|}
\hline ID & Name & Output-End Form & Scale & Year & Related Study \\
\hline 1 & JOBS & Prolog-style Queries & 640 & 1996 & $3,11,50$ \\
\hline 2 & GEO & Lambda Calculus / SQL & 880 & 1996 & $\begin{array}{c}3,10,17,23,24,28,32 \\
41,50,54,57,60,65\end{array}$ \\
\hline 3 & SAIL & Custom Actions & 3236 & 2006 & 28 \\
\hline 4 & ATIS & Lambda Calculus / SQL & 5871 & 2010 & $\begin{array}{c}8,10,11,17,23,24,32,37 \\
41,46,50,57,58,60,61\end{array}$ \\
\hline 5 & WEBQUESTIONS & Lambda DCS & 5810 & 2013 & $29,37,44,54$ \\
\hline 6 & IFTTT 2015 & If-This-Then-That Recipes & 86,960 & 2015 & $2,5,13,25$ \\
\hline 7 & DJANGO & Python & 18,805 & 2015 & $1,2,8,10,12,17,65$ \\
\hline 8 & OverNight & Lambda DCS & 13,682 & 2015 & $38,39,49,60,63$ \\
\hline 9 & MTG & Java & 13,297 & 2016 & 1 \\
\hline 10 & HS & Python & 665 & 2016 & $\begin{array}{c}1,2,3,11,12 \\
18,22,23\end{array}$ \\
\hline 11 & WikiTableQuestions & Lambda DCS & 222,033 & 2016 & 4,59 \\
\hline 12 & IFTTT 2016 & If-This-Then-That Recipes & 291,285 & 2016 & 25,48 \\
\hline 13 & NLMAPS & Lambda Calculus & 2380 & 2016 & 28 \\
\hline 14 & SCENE & Custom Actions & 4403 & 2016 & 30 \\
\hline 15 & ALCHEMY & Custom Actions & 4560 & 2016 & 30 \\
\hline 16 & TANGRAMS & Custom Actions & 4989 & 2016 & 30 \\
\hline 17 & NL-RX & Regular Expression & 10,000 & 2016 & 31 \\
\hline 18 & SPADES & Lambda Calculus & 79,247 & 2016 & 37,54 \\
\hline 19 & GRAPHQUESTIONS & Graph Query & 5166 & 2016 & $37,40,54$ \\
\hline 20 & WikiSQL & SQL & 80,654 & 2017 & $\begin{array}{c}7,10,21,33,34,36 \\
42,55,57,59,62\end{array}$ \\
\hline 21 & WEBQUESTIONSSP & lambda DCS & 4737 & 2016 & 14 \\
\hline 22 & SENLIDB & SQL & 24,890 & 2017 & 21 \\
\hline 23 & Scholar & SQL & 817 & 2017 & 32 \\
\hline 24 & Textbook & SQl & 108 & 2017 & 35 \\
\hline 25 & NL-Bash & Bash & 5413 & 2017 & 40 \\
\hline 26 & AML & AML & 150,000 & 2018 & 6 \\
\hline 27 & ALGOLISP & DSL inspired by LISP & 100,000 & 2018 & 9,15 \\
\hline 28 & CONALA & Python & 2879 & 2018 & 17 \\
\hline 29 & Advising & SQL & 4385 & 2018 & 24 \\
\hline 30 & Karel & Customer Tree & 10,000 & 2018 & 43 \\
\hline 31 & CONCODE & Java & 100,000 & 2018 & $19,45,46$ \\
\hline 32 & Spider & SQL & 10,181 & 2018 & $18,47,51,56,58,66$ \\
\hline 33 & ComplexWebQuestions & SPARQL & 34,689 & 2018 & 52 \\
\hline 34 & GeoGrAnno & lambda calculus & 824 & 2019 & 63 \\
\hline 35 & ACBJ & Java & $1,074,963$ & 2019 & 64 \\
\hline 36 & MIMICSQL & SQL & 10,000 & 2020 & 53 \\
\hline
\end{tabular}

\section{References}

1. Mur, R.A. Automatic inductive programming. In Proceedings of the 23rd International Conference on Machine Learning, Tutorial, Pittsburgh, PA, USA, 25-29 June 2006.

2. Hu, X.; Li, G.; Xia, X.; Lo, D.; Jin, Z. Deep code comment generation. In Proceedings of the 2018 IEEE/ACM 26th International Conference on Program Comprehension (ICPC), Gothenburg, Sweden, 27-28 May 2018; pp. 200-20010.

3. Gu, X.; Zhang, H.; Kim, S. Deep code search. In Proceedings of the 40th International Conference on Software Engineering, Gothenburg, Sweden, 27 May-3 June 2018; pp. 933-944.

4. Rabinovich, M.; Stern, M.; Klein, D. Abstract syntax networks for code generation and semantic parsing. arXiv 2017, arXiv:1704.07535. 
5. $\quad$ Ling, W.; Blunsom, P.; Grefenstette, E.; Hermann, K.M.; Kočiský, T.; Wang, F.; Senior, A. Latent predictor networks for code generation. arXiv 2016, arXiv:1603.06744.

6. Yin, P.; Neubig, G. Reranking for neural semantic parsing. In Proceedings of the 57th Annual Meeting of the Association for Computational Linguistics, Florence, Italy, 28 July-2 August 2019; pp. 4553-4559.

7. Shin, E.C.; Allamanis, M.; Brockschmidt, M. Program synthesis and semantic parsing with learned code idioms. In Proceedings of the Advances in Neural Information Processing Systems, Vancouver, BC, Canada, 8-14 December 2019; pp. 10825-10835.

8. Bednarek, J.; Piaskowski, K.; Krawiec, K. Ain't Nobody Got Time For Coding: Structure-Aware Program Synthesis from Natural Language. arXiv 2018, arXiv:1810.09717.

9. Polosukhin, I.; Skidanov, A. Neural program search: Solving programming tasks from description and examples. arXiv 2018, arXiv:1802.04335.

10. Liang, C.; Berant, J.; Le, Q.; Forbus, K.D.; Lao, N. Neural symbolic machines: Learning semantic parsers on freebase with weak supervision. arXiv 2016, arXiv:1611.00020.

11. Guan, W.; Liu, Q.; Han, G.; Wang, B.; Li, S. An Improved Coarse-to-Fine Method for Solving Generation Tasks. In Proceedings of the 17th Annual Workshop of the Australasian Language Technology Association, Sydney, Australia, 4-6 December 2019; Australasian Language Technology Association: Sydney, Australia; pp. 178-185.

12. Lin, K.; Bogin, B.; Neumann, M. Grammar-based neural text-to-sql generation. arXiv 2019, arXiv:1905.13326.

13. Yin, P.; Neubig, G. A syntactic neural model for general-purpose code generation. arXiv 2017, arXiv:1704.01696.

14. Murali, V.; Qi, L.; Chaudhuri, S.; Jermaine, C. Neural sketch learning for conditional program generation. arXiv 2017, arXiv:1703.05698.

15. Lee, D.; Yoon, J.; Song, J.; Lee, S.; Yoon, S. One-shot learning for text-to-sql generation. arXiv 2019, arXiv:1905.11499.

16. Bunel, R.; Hausknecht, M.; Devlin, J.; Singh, R.; Kohli, P. Leveraging grammar and reinforcement learning for neural program synthesis. arXiv 2018, arXiv:1805.04276.

17. Wang, C.; Tatwawadi, K.; Brockschmidt, M. Robust text-to-sql generation with execution-guided decoding. arXiv 2018, arXiv:1807.03100.

18. Hayati, S.A.; Olivier, R.; Avvaru, P. Retrieval-based neural code generation. arXiv 2018, arXiv:1808.10025.

19. Sun, Z.; Zhu, Q.; Mou, L.; Xiong, Y.; Li, G.; Zhang, L. A grammar-based structural cnn decoder for code generation. In Proceedings of the AAAI Conference on Artificial Intelligence, Honolulu, HI, USA, 27 January-1 February 2019; Volume 33, pp. 7055-7062.

20. Sun, Z.; Zhu, Q.; Xiong, Y.; Sun, Y.; Mou, L.; Zhang, L. TreeGen: A Tree-Based Transformer Architecture for Code Generation. In Proceedings of the AAAI, New York, NY, USA, 7-12 February 2020; pp. 8984-8991.

21. Iyer, S.; Cheung, A.; Zettlemoyer, L. Learning programmatic idioms for scalable semantic parsing. arXiv 2019, arXiv:1904.09086.

22. Zeng, Y.; Gao, Y.; Guo, J.; Chen, B.; Liu, Q.; Lou, J.-G.; Teng, F.; Zhang, D. RECPARSER: A Recursive Semantic Parsing Framework for Text-to-SQL Task. Available online: https:/ /www.ijcai.org/Proceedings/2020/0504.pdf (accessed on 20 August 2021).

23. Shi, T.; Tatwawadi, K.; Chakrabarti, K. Incsql: Training incremental text-to-sql parsers with non-deterministic oracles. arXiv 2019, arXiv:1809.05054.

24. Xu, X.; Liu, C.; Song, D. Sqlnet: Generating structured queries from natural language without reinforcement learning. arXiv 2017, arXiv:1711.04436.

25. Hu, H.; Chen, Q.; Liu, Z. Code Generation from Supervised Code Embeddings. In Proceedings of the International Conference on Neural Information Processing; Springer: Cham, Switzerland, 2019; pp. 388-396.

26. Dong, L.; Lapata, M. Coarse-to-fine decoding for neural semantic parsing. arXiv 2018, arXiv:1805.04793.

27. Xu, K.; Wu, L.; Wang, Z.; Yu, M.; Chen, L.; Sheinin, V. Exploiting rich syntactic information for semantic parsing with graph-tosequence model. arXiv 2018, arXiv:1808.07624.

28. Stehnii, A. Generation of Code from Text Description with Syntactic Parsing and Tree2Tree Model. 2018. Available online: https: / / er.ucu.edu.ua/handle/1/1191 (accessed on 20 August 2021).

29. Liu, C.; Chen, X.; Shin, E.C.; Chen, M.; Song, D. Latent attention for if-then program synthesis. In Proceedings of the Advances in Neural Information Processing Systems, Barcelona, Spain, 5-10 December 2016; pp. 4574-4582.

30. Zhang, H.; Cai, J.; Xu, J.; Wang, J. Complex question decomposition for semantic parsing. In Proceedings of the 57th Annual Meeting of the Association for Computational Linguistics, Florence, Italy, 28 July-2 August 2019; pp. 4477-4486.

31. Brad, F.; Iacob, R.; Hosu, I.; Ruseti, S.; Rebedea, T. A Syntax-Guided Neural Model for Natural Language Interfaces to Databases. In Proceedings of the 2018 IEEE 30th International Conference on Tools with Artificial Intelligence (ICTAI), Volos, Greece, 5-7 November 2018; pp. 229-233.

32. Iyer, S.; Konstas, I.; Cheung, A.; Krishnamurthy, J.; Zettlemoyer, L. Learning a neural semantic parser from user feedback. arXiv 2017, arXiv:1704.08760.

33. Dong, L.; Lapata, M. Language to logical form with neural attention. arXiv 2016, arXiv:1601.01280.

34. Yu, T.; Yasunaga, M.; Yang, K. Syntaxsqlnet: Syntax tree networks for complex and cross-domaintext-to-sql task. arXiv 2018, arXiv:1810.05237.

35. Wang, P.; Shi, T.; Reddy, C. Text-to-SQL Generation for Question Answering on Electronic Medical Records. In Proceedings of the Web Conference 2020, Taipei, Taiwan, 20-24 April 2020; pp. 350-361.

36. Yaghmazadeh, N.; Wang, Y.; Dillig, I.; Dillig, T. SQLizer: Query synthesis from natural language. Proc. ACM Program. Lang. 2017, 1, 1-26. [CrossRef] 
37. Huang, P.S.; Wang, C.; Singh, R. Natural language to structured query generation via meta-learning. arXiv 2018, arXiv:1803.02400.

38. Fan, X.; Monti, E.; Mathias, L.; Dreyer, M.; Blunsom, P.; Bordes, A.; Cho, K.; Cohen, S.; Dyer, C.; Grefenstette, E.; et al. Transfer learning for neural semantic parsing. arXiv 2017, arXiv:1706.04326.

39. Yin, P.; Neubig, G. Tranx: A transition-based neural abstract syntax parser for semantic parsing and code generation. arXiv 2018, arXiv:1810.02720.

40. Locascio, N.; Narasimhan, K.; De León, E.; Kushman, N.; Barzilay, R. Neural generation of regular expressions from natural language with minimal domain knowledge. arXiv 2016, arXiv:1608.03000.

41. Beltagy, I.; Quirk, C.; Erk, K.; Smith, N.A. Improved semantic parsers for if-then statements. In Proceedings of the 54th Annual Meeting of the Association for Computational Linguistics (Volume 1: Long Papers), Berlin, Germany, 7-12 August 2016; pp. 726-736.

42. Shin, R. Encoding database schemas with relation-aware self-attention for text-to-sql parsers. arXiv 2019, arXiv:1906.11790.

43. Sun, Y.; Tang, D.; Duan, N.; Ji, J.; Cao, G.; Feng, X.; Qin, B.; Liu, T.; Zhou, M. Semantic parsing with syntax-and table-aware sql generation. arXiv 2018, arXiv:1804.08338.

44. Inan, H.A.; Tomar, G.S.; Pan, H. Improving Semantic Parsing with Neural Generator-Reranker Architecture. arXiv 2019, arXiv:1909.12764..

45. Campagna, G.; Xu, S.; Moradshahi, M.; Socher, R.; Lam, M.S. Genie: A generator of natural language semantic parsers for virtual assistant commands. In Proceedings of the 40th ACM SIGPLAN Conference on Programming Language Design and Implementation, Phoenix, AZ, USA, 22-26 June 2019; pp. 394-410.

46. Guo, D.; Tang, D.; Duan, N.; Zhou, M.; Yin, J. Coupling Retrieval and Meta-Learning for Context-Dependent Semantic Parsing. arXiv 2019, arXiv:1906.07108.

47. Wang, C.; Brockschmidt, M.; Singh, R. Pointing out SQL Queries from Text. 2018. arXiv 2017, arXiv:1709.00103. Available online: https:/ / openreview.net/forum?id=BkUDW_1Cb (accessed on 20 August 2021).

48. Guu, K.; Pasupat, P.; Liu, E.; Liang, P.; Barzilay, R.; Kan, M.-Y. From language to programs: Bridging reinforcement learning and maximum marginal likelihood. arXiv 2017, arXiv:1704.07926.

49. Cheng, J.; Lapata, M. Weakly-supervised neural semantic parsing with a generative ranker. arXiv 2018, arXiv:1808.07625.

50. Wang, B.; Titov, I.; Lapata, M. Learning Semantic Parsers from Denotations with Latent Structured Alignments and Abstract Programs. arXiv 2019, arXiv:1909.04165.

51. Kočiský, T.; Melis, G.; Grefenstette, E.; Dyer, C.; Ling, W.; Blunsom, P.; Hermann, K.M. Semantic parsing with semi-supervised sequential autoencoders. arXiv 2019, arXiv:1609.09315.

52. Cao, R.; Zhu, S.; Yang, C.; Liu, C.; Ma, R.; Zhao, Y.; Chen, L.; Yu, K. Unsupervised Dual Paraphrasing for Two-stage Semantic Parsing. arXiv 2020, arXiv:2005.13485.

53. Yin, P.; Zhou, C.; He, J.; Neubig, G. StructVAE: Tree-structured latent variable models for semi-supervised semantic parsing. arXiv 2018, arXiv:1806.07832.

54. Tang, X.; Wang, Z.; Qi, J.; Li, Z. Improving Code Generation From Descriptive Text By Combining Deep Learning and Syntax Rules. In Proceedings of the SEKE, Lisbon, Portugal, 10-12 July 2019; pp. 385-496.

55. Zhong, V.; Xiong, C.; Socher, R. Seq2sql: Generating structured queries from natural language using reinforcement learning. arXiv 2017, arXiv:1709.00103.

56. Cheng, J.; Reddy, S.; Saraswat, V.; Lapata, M.; Barzilay, R.; Kan, M.-Y. Learning structured natural language representations for semantic parsing. arXiv 2017, arXiv:1704.08387.

57. Jia, R.; Liang, P. Data recombination for neural semantic parsing. arXiv 2016, arXiv:1606.03622.

58. Yu, T.; Li, Z.; Zhang, Z.; Zhang, R.; Radev, D. Typesql: Knowledge-based type-aware neural text-to-sql generation. arXiv 2018, arXiv:1804.09769.

59. Krishnamurthy, J.; Dasigi, P.; Gardner, M. Neural Semantic Parsing with Type Constraints for Semi-Structured Tables. In Proceedings of the 2017 Conference on Empirical Methods in Natural Language Processing, Copenhagen, Denmark, 7-11 September 2017; pp. 1516-1526.

60. Chaurasia, S.; Mooney, R. Dialog for language to code. In Proceedings of the Eighth International Joint Conference on Natural Language Processing (Volume 2: Short Papers), Taipei, Taiwan, 27 November-1 December 2017; pp. 175-180.

61. Iyer, S.; Konstas, I.; Cheung, A.; Zettlemoyer, L. Mapping language to code in programmatic context. arXiv 2018, arXiv:1808.09588.

62. Yih, S.W.T.; Chang, M.W.; He, X.; Gao, J. Semantic Parsing via Staged Query Graph Generation: Question Answering with Knowledge Base. 2015. Available online: https://www.microsoft.com/en-us/research/publication/semantic-parsing-viastaged-query-graph-generation-question-answering-with-knowledge-base/ (accessed on 20 August 2021).

63. Lin, X.V.; Wang, C.; Pang, D.; Vu, K.; Ernst, M.D. Program Synthesis from Natural Language Using Recurrent Neural Networks; Tech. Rep. UW-CSE-17-03-01; University of Washington Department of Computer Science and Engineering: Seattle, WA, USA, 2017.

64. Herzig, J.; Berant, J. Decoupling structure and lexicon for zero-shot semantic parsing. arXiv 2018, arXiv:1804.07918.

65. Su, Y.; Yan, X. Cross-domain semantic parsing via paraphrasing. arXiv 2017, arXiv:1704.05974.

66. Yao, Z.; Li, X.; Gao, J.; Sadler, B.; Sun, H. Interactive semantic parsing for if-then recipes via hierarchical reinforcement learning. In Proceedings of the AAAI Conference on Artificial Intelligence, Honolulu, HI, USA, 27 January-1 February 2019; Volume 33, pp. 2547-2554. 
67. Chen, B.; Sun, L.; Han, X. Sequence-to-action: End-to end semantic graph generation for semantic parsing. arXiv 2018, arXiv:1809.00773.

68. Cheng, J.; Reddy, S.; Saraswat, V.; Lapata, M. Learning an executable neural semantic parser. Comput. Linguist. 2019, 45, 59-94. [CrossRef]

69. Huo, S.; Ma, T.; Chen, J.; Chang, M.; Wu, L.; Witbrock, M.J. Graph Enhanced Cross-Domain Text-to-SQL Generation. In Proceedings of the Thirteenth Workshop on Graph-Based Methods for Natural Language Processing (TextGraphs-13), Hong Kong, China, 4 November 2019; pp. 159-163.

70. Adam, S.; Larsen, M.; Jensen, K.; Schultz, U.P. Towards Rule-Based Dynamic Safety Monitoring for Mobile Robots. Lect. Notes Comput. Sci. 2016, 88, 207-218.

71. Cataño, N.; Rivera, V. EventB2Java: A Code Generator for Event-B; NASA Formal Methods; Springer International Publishing: Berlin/Heidelberg, Germany, 2016.

72. Koehler, J.; Hauser, R.; Sendall, S.; Wahler, M. Declarative techniques for model-driven business process integration. IBM Syst. J. 2005, 44, 47-65. [CrossRef]

73. Viswanathan, S.E.; Samuel, P. Automatic code generation using unified modeling language activity and sequence models. IET Software 2016, 10, 164-172. [CrossRef]

74. Philippi, S. Automatic code generation from high-level Petri-Nets for model driven systems engineering. J. Syst. Softw. 2006, 79, 1444-1455. [CrossRef]

75. Chen, C.; Su, T.; Meng, G.; Xing, Z.; Liu, Y. From UI design image to GUI skeleton: A neural machine translator to bootstrap mobile GUI implementation. In Proceedings of the 40th International Conference on Software Engineering, Gothenburg, Sweden, 27 May-3 June 2018; pp. 665-676.

76. Wohlin, C. Guidelines for Snowballing in Systematic Literature Studies and a Replication in Software Engineering. In Proceedings of the 18th International Conference on Evaluation and Assessment in Software Engineering, London, UK, 13-14 May 2014; pp. $1-10$.

77. Bahdanau, D.; Cho, K.; Bengio, Y. Neural machine translation by jointly learning to align and translate. arXiv 2014, arXiv:1409.0473.

78. Barendregt, H.P. The Lambda Calculus; North-Holland: Amsterdam, The Netherlands, 1984.

79. Liang, P.; Jordan, M.; Klein, D. Learning Dependency-Based Compositional Semantics. Comput. Linguist. 2013, 39, 389-446._a_00127. [CrossRef]

80. Allamanis, M.; Barr, E.T.; Devanbu, P.; Sutton, C. A Survey of Machine Learning for Big Code and Naturalness. ACM Comput. Surv. 2018, 51, 1-37. [CrossRef]

81. Alur, R.; Bodik, R.; Juniwal, G.; Martin, M.M.; Raghothaman, M.; Seshia, S.A.; Singh, R.; Solar-Lezama, A.; Torlak, E.; Udupa, A.; et al. Syntax-Guided Synthesis; IEEE: New York, NY, USA, 2013.

82. Kalyanpur, A.; Patwardhan, S.; Boguraev, B.K.; Lally, A.; Chu-Carroll, J. Fact-based question decomposition in DeepQA. IBM J. Res. Dev. 2012, 56, 13. [CrossRef]

83. Pazos, R.; Rodolfo, A.; Juan, J.; González, B.; Marco, A.; Aguirre, L.; José, A.; Martínez, F.;Héctor, J.; Fraire, H. Natural language interfaces to databases: An analysis of the state of the art. In Recent Advances on Hybrid Intelligent Systems; Springer: Berlin/Heidelberg, Germany, 2013; pp. 463-480.

84. Wang, Y.; Berant, J.; Liang, P. Building a semantic parser overnight. In Proceedings of the 53rd Annual Meeting of the Association for Computational Linguistics and the 7th International Joint Conference on Natural Language Processing (Volume 1: Long Papers), Beijing, China, 26-31 July 2015; pp. 1332-1342.

85. Kushman, N.; Barzilay, R. Using semantic unification to generate regular expressions from natural language. In Proceedings of the 2013 Conference of the North American Chapter of the Association for Computational Linguistics: Human Language Technologies, Atlanta, GA, USA, 9-14 June 2013; pp. 826-836.

86. A Recipe for Training Neural Networks. Available online: http://karpathy.github.io/2019/04/25/recipe/ (accessed on 5 September 2020).

87. Khassanov, Y.; Zeng, Z.; Pham, V.T.; Xu, H.; Chng, E.S. Enriching Rare Word Representations in Neural Language Models by Embedding Matrix Augmentation. arXiv 2019, arXiv:1904.03799.

88. Kamath, A.; Das, R. A survey on semantic parsing. arXiv 2018, arXiv:1812.00978.

89. Zhang, J.; Utiyama, M.; Sumita, E.; Neubig, G.; Nakamura, S. Guiding neural machine translation with retrieved translation pieces. arXiv 2018, arXiv:1804.02559. 